OPEN ACCESS

Edited by:

Stefania Zanetti,

University of Sassari, Italy

Reviewed by:

Márió Gajdács,

University of Szeged, Hungary

Payam Behzadi,

Islamic Azad University, ShahreQods,

Iran

*Correspondence:

Jesús Campos-García

jesus.campos@umich.mx;

jcampos_garcia70@yahoo.com orcid.org/0000-0002-8337-5830

Specialty section:

This article was submitted to Antimicrobials, Resistance

and Chemotherapy,

a section of the journa

Frontiers in Microbiology

Received: 28 September 2021

Accepted: 19 October 2021

Published: 16 November 2021

Citation:

Martínez-Alcantar L, Orozco G,

Díaz-Pérez AL, Villegas J,

Reyes-De la Cruz H, García-Pineda E

and Campos-García J (2021)

Participation of Acyl-Coenzyme

A Synthetase FadD4 of Pseudomonas

aeruginosa PAO1 in Acyclic

Terpene/Fatty Acid Assimilation

and Virulence by Lipid A Modification.

Front. Microbiol. 12:785112.

doi: 10.3389/fmicb.2021.785112

\section{Participation of Acyl-Coenzyme A Synthetase FadD4 of Pseudomonas aeruginosa PAO1 in Acyclic Terpene/Fatty Acid Assimilation and Virulence by Lipid A Modification}

\author{
Lorena Martínez-Alcantar', Gabriela Orozco', Alma Laura Díaz-Pérez', Javier Villegas², \\ Homero Reyes-De la Cruz ${ }^{3}$, Ernesto García-Pineda ${ }^{4}$ and Jesús Campos-García ${ }^{\text {* }}$ \\ ${ }^{1}$ Laboratorio de Biotecnología Microbiana, Instituto de Investigaciones Químico Biológicas, Universidad Michoacana de San \\ Nicolás de Hidalgo, Morelia, Mexico, ${ }^{2}$ Laboratorio de Interacción Suelo, Planta, Microorganismo, Instituto \\ de Investigaciones Químico Biológicas, Universidad Michoacana de San Nicolás de Hidalgo, Morelia, Mexico, ${ }^{3}$ Laboratorio \\ de Control Traduccional, Instituto de Investigaciones Químico Biológicas, Universidad Michoacana de San Nicolás \\ de Hidalgo, Morelia, Mexico, ${ }^{4}$ Laboratorio de Bioquímica y Biología Molecular, Instituto de Investigaciones Químico \\ Biológicas, Universidad Michoacana de San Nicolás de Hidalgo, Morelia, Mexico
}

The pathogenic bacterium Pseudomonas aeruginosa possesses high metabolic versatility, with its effectiveness to cause infections likely due to its well-regulated genetic content. P. aeruginosa PAO1 has at least six fadD paralogous genes, which have been implicated in fatty acid (FA) degradation and pathogenicity. In this study, we used mutagenesis and a functional approach in $P$. aeruginosa PAO1 to determine the roles of the fadD4 gene in acyclic terpene (AT) and FA assimilation and on pathogenicity. The results indicate that fadD4 encodes a terpenoyl-CoA synthetase utilized for AT and FA assimilation. Additionally, mutations in fadD paralogs led to the modification of the quorum-sensing las/rh/ systems, as well as the content of virulence factors pyocyanin, biofilm, rhamnolipids, lipopolysaccharides (LPS), and polyhydroxyalkanoates. In a Caenorhabditis elegans in vivo pathogenicity model, culture supernatants from the 24-h-grown fadD4 single mutant increased lethality compared to the PAO1 wild-type (WT) strain; however, the double mutants fadD1/fadD2, fadD1/fadD4, and fadD2/fadD4 and single mutant fadD2 increased worm survival. A correlation analysis indicated an interaction between worm death by the PAO1 strain, the fadD4 mutation, and the virulence factor LPS. Fatty acid methyl ester (FAME) analysis of LPS revealed that a proportion of the LPS and FA on lipid A were modified by the fadD4 mutation, suggesting that FadD4 is also involved in the synthesis/degradation and modification of the lipid A component of LPS. LPS isolated from the fadD4 mutant and double mutants fadD1/fadD4 and fadD2/fadD4 showed a differential behavior to induce an increase in body temperature in rats injected with LPS compared to the WT strain or from the fadD1 and fadD2 mutants. In agreement, LPS isolated from the fadD4 mutant and double mutants fadD1/fadD2 and fadD2/fadD4 increased the induction of IL-8 in rat sera, but IL1- $\beta$ cytokine levels decreased in the double mutants fadD1/fadD2 and fadD1/fadD4. The results indicate that the $f a d D$ genes are implicated in the degree of pathogenicity of 
P. aeruginosa PAO1 induced by LPS-lipid A, suggesting that FadD4 contributes to the removal of acyl-linked FA from LPS, rendering modification in its immunogenic response associated to Toll-like receptor TLR4. The genetic redundancy of fadD is important for bacterial adaptability and pathogenicity over the host.

Keywords: acyl-CoA synthetase, acyclic terpenes, fatty acids, host colonization, lipopolysaccharides, polyhydroxyalkanoates, biofilm

\section{INTRODUCTION}

Pseudomonas aeruginosa is considered pathogenic to humans, insects, nematodes, and animals and possesses high metabolic versatility among bacteria capable of growing on a wide variety of organic compounds (Valentini et al., 2018; Moser et al., 2021). The effectiveness of this species in causing infection and its metabolic versatility may be attributed to its well-regulated genetic content and the accumulation of novel orthologous and paralogous genes in its genome, resulting in genetic redundancy (Stover et al., 2000; Toll-Riera et al., 2016; Panayidou et al., 2020). P. aeruginosa is an opportunistic human pathogen capable of causing a wide array of life-threatening acute and chronic infections, particularly in patients with compromised immune defenses. It is the main cause of morbidity and mortality in patients with cystic fibrosis and one of the leading nosocomial pathogens affecting hospitalized patients (Moradali et al., 2017; Rossi et al., 2021). P. aeruginosa employs several mechanisms to overcome host defenses, including the production of a wide range of virulence factors that allow its colonization and adaptation in the host, which are finely regulated and dependent on cell-tocell communication (Lee and Zhang, 2015; Behzadi et al., 2021a; Moser et al., 2021).

The FadD proteins (fatty acid coenzyme A synthetases) involved in fatty acid degradation (FAD) are extensively distributed across all kingdoms of life. Recently, multiple homologs have been identified also as virulence factors in several bacteria such as Mycobacterium tuberculosis, Salmonella enterica serovar Typhimurium, and Haemophilus parasuis; however, its genetic redundancy is uncertain in terms of bacterial pathogenicity (Kang et al., 2010; Feng et al., 2017). In $P$. aeruginosa PAO1, two acyl-CoA synthetases (FadD1 and FadD2) are involved in FA activation, contributing to differential FA degradation and bacterial virulence (Kang et al., 2010). FadD1 has a preference for linear long-chain $\mathrm{FA}\left(\mathrm{C}_{6}-\right.$ $\left.\mathrm{C}_{14}\right)$ and FadD2 for short- and medium-chain FA $\left(\mathrm{C}_{4}-\mathrm{C}_{10}\right)$. Additionally, other FadD homologs have been identified in $P$. aeruginosa, rendering up to six $f a d D$ genes (fadD1-D6) related to FA degradation (Zarzycki-Siek et al., 2013). The fadD1 and fadD2 genes have been linked to virulence through the degradation of phosphatidylcholine (the main component of the lung surfactant), but without causing modifications in the production of virulence factors such as proteases, lipases, and phospholipases (Zarzycki-Siek et al., 2013).

Pseudomonas aeruginosa $f a d D 1$ and $f a d D 2$ are the main genes involved in FA degradation, and fadD4 (ORF PA1617) is related to acyclic terpene (AT) utilization (Zarzycki-Siek et al., 2013). The ability of P. aeruginosa to assimilate acyclic terpenes and leucine/isovalerate as sole carbon and energy sources is well known (Diaz-Perez et al., 2004; Aguilar et al., 2006; Forster-Fromme et al., 2006; Chavez-Aviles et al., 2009; Campos-García, 2010). The acyclic terpene catabolic pathway is considered to consist of four steps: (i) upper oxidation activation pathway, (ii) central AT pathway, (iii) $\beta$-oxidation coupling, and (iv) convergence with the leucine/isovalerate pathway (Campos-García, 2010). The genes involved in the AT and leucine/isovalerate pathways are present in the atuRABCDEFGH and liuRABCDE clusters, respectively; their encoded enzymes have been characterized at the biochemical level, and for a majority of genes, their function in the catabolic pathway is demonstrated (Campos-García, 2010; Díaz-Pérez et al., 2015; Diaz-Perez et al., 2018). The upper pathway of AT degradation involves the oxidation of terpenes such as citronellol, geraniol, or nerol to citronellic and geranic acids (CamposGarcía and Soberón-Chávez, 2000; Forster-Fromme et al., 2006; Campos-García, 2010). The citronellic or geranic acids produced are then activated by a putative acyl-CoA synthetase, producing citronellyl-CoA or geranyl-CoA, respectively; the corresponding gene remains unknown. The citronellyl-CoA and trans-geranylCoA metabolites produced in the preceding step are oxidized or isomerized to cis-geranyl-CoA, the starting metabolite of the central AT pathway. Therefore, it is suggested that terpenoyl-CoA synthesis is carried out by ATP-dependent acyl-CoA synthetase (Campos-García, 2010). The atuH gene has been suggested to encode the acyl-CoA synthase involved in terpenoyl activation (Forster-Fromme et al., 2006); however, this has not been completely clarified.

Fatty acids are important metabolic intermediates and phospholipid components, which are essential for membrane formation. In addition to the energy-expensive FA biosynthesis, most pathogens use and incorporate extracellular FA into their membrane phospholipids and lipopolysaccharides (LPS) (Feng et al., 2017). FadD paralog proteins (FadD1 to FadD6) have been described to be involved in the degree of pathogenesis and virulence mechanism of $P$. aeruginosa PAO1 in a lung mouse infection model, evaluating the modification in the production of virulence factors (such as protease, hemolysin, lipases, phospholipases, and rhamnolipids) (Kang et al., 2010; Zarzycki-Siek et al., 2013).

Multiple virulence factors have been described as inducers or the innate immune responses mediated by polymorphonuclear and macrophage cells (Moser et al., 2021). In the mammalian innate immune responses constituted by leukocyte type-dependent response via Toll-like receptors (TLRs), at least 13 TLRs have been identified in mice and humans (McIsaac et al., 2012). TLRs are activated through 
binding to damage/danger-associated molecular patterns (DAMPs), microbial/microbe-associated molecular patterns (MAMPs), pathogen-associated molecular patterns (PAMPs), and xenobiotic-associated molecular patterns (XAMPs) (Behzadi et al., 2021b).

Toll-like receptors play essential roles in host defense against Gram-negative bacteria (McIsaac et al., 2012; Moser et al., 2021). Thus, TLR1-6 receptors have been associated with the mechanism of recognition of PAMPs such as LPS, flagellin, lipoproteins, pilus, peptidoglycans, etc. in P. aeruginosa (Behzadi et al., 2021b; Moser et al., 2021). For example, the TLR2 contributes in the induction of excessive inflammation produced in response to ligands such as lipoproteins, components of the extracellular capsule, and secreted toxins, while that TLR5 response is associated to flagellin. The pathogenesis of $P$. aeruginosa is associated to TLR4 recognizing LPS by binding to the lipid A component on the outer membrane of bacteria (McIsaac et al., 2012; Behzadi et al., 2021b). LPS, as an integral component of the $P$. aeruginosa cell envelope, is important for bacterium-host interactions and has been shown to be a major virulence factor (Lam et al., 2011). In $P$. aeruginosa, the cell surface carbohydrate polymer known as B-band O-antigen, which is a component of LPS, is a major virulence factor, and a wide variety of serotypes exist that differ in the structure of the O-antigen (King et al., 2008; Lam et al., 2011; Lo Sciuto et al., 2019). Genetic determinants have been well characterized, and their involvement in bacterial pathogenicity has been studied; serotype $\mathrm{O} 12$ is associated with virulence, multidrug resistance, cystic fibrosis patients, and burn wounds (Pier, 2007; Moradali et al., 2017; Lo Sciuto et al., 2019). Over 50 genes in the $P$. aeruginosa PAO1 genome database are associated with LPS biosynthesis; these clusters are mainly related to antigen $\mathrm{O}$ and the core of polysaccharides, but genetic determinants associated with lipid A are poorly described $^{1}$. Some studies describe in cystic fibrosis patients that $P$. aeruginosa produces several different varieties of lipid A on LPS, contributing to the degree of virulence, which is associated to hexa-acylated lipid A, the more potent agonist to TLR4 receptor (McIsaac et al., 2012).

Therefore, the role of FadD proteins in the pathogenicity mechanisms of $P$. aeruginos $a$ and its relation with virulence factor production are of great interest. In this study, a gene mutagenesis and functional approach in $P$. aeruginosa PAO1 were used to determine the roles of $f a d D$ genes in acyclic terpene and fatty acid catabolism and their contribution to bacterial pathogenicity related with the LPS virulence factor and the immunological response induced.

\section{MATERIALS AND METHODS}

\section{Bacterial Strains, Plasmids, and Culture Conditions}

Bacterial strains and plasmids used in this work are shown in Table 1. The strains were grown at $30^{\circ} \mathrm{C}$ in Luria broth (LB) or

${ }^{1}$ www.pseudomonas.com
M9 minimal medium (Sambrook, 2001). For rhamnolipid (RHL) and polyhydroxyalkanoate (PHA) production, $P$. aeruginosa strains were cultured in phosphate-limited protease peptone glucose ammonium salt (PPGAS) medium using glucose as the main carbon source. Solid media were prepared by adding $1.5 \%$ agar. Antibiotic concentrations used for $P$. aeruginosa strains were as follows: streptomycin (Sm) $200 \mu \mathrm{g} / \mathrm{ml}$, gentamicin (Gm) $80 \mu \mathrm{g} / \mathrm{ml}$, and tetracycline (Tc) $60 \mu \mathrm{g} / \mathrm{ml}$; for Escherichia coli strains, ampicillin (Ap) $100 \mu \mathrm{g} / \mathrm{ml}, \mathrm{Gm} 20 \mu \mathrm{g} / \mathrm{ml}$, and Tc $15 \mu \mathrm{g} / \mathrm{ml}$. Strains were tested for their ability to grow on M9 agar plates supplemented with citronellol, citronellic acid, isovaleric acid (Merck), palm fatty acids, or phosphatidylcholine as sole carbon and energy source after an incubation of $48 \mathrm{~h}$ at $30^{\circ} \mathrm{C}$.

\section{Cloning, Expression, and Purification of Recombinant Proteins}

The nucleotide and amino acid sequences analyzed are denoted in the Pseudomonas Genome Project (see Text Footnote 1) as ORFs PA3299, PA3300, and PA1617 (Stover et al., 2000), which were named as fadD1, fadD2, and fadD4 genes, respectively (Zarzycki-Siek et al., 2013). The fadD4 gene was amplified by PCR using PAO1 genomic DNA as template with Platinum Pfx DNA Polymerase (Invitrogen) according to the manufacturer's instructions. Primers were designed from the PAO1 genome sequence, fadD4-forward $5^{\prime}$ GGATCCCATGGTCACTGCAAATCGTCTGCCG $3^{\prime}$ and fadD4-reverse 5'AAGCTTTTCGTG CCAGAGCACGGCCTC3' with the insertion of the restriction sites BamHI and HindIII. The PCR fragment obtained ( $\sim 1.7 \mathrm{kbp})$ was cloned into the pGEM$\mathrm{T}$ Easy vector to give the plasmid pGfadD4. Next, the fadD4 gene was removed using BamHI and HindIII endonucleases (Invitrogen), purified, and ligated into the pCDFDuet-1 vector to obtain the pCDfadD4 plasmid (Table 1). This plasmid was designed to merge a $6 \times$-histidine tag into the $\mathrm{N}$-terminal end of the FadD4 protein. The plasmids were introduced by electroporation to E. coli JM101 cells and subsequently analyzed by digestion with restriction endonucleases and confirmed by DNA sequencing.

Plasmid-transformed E. coli JM101 strain was cultured in $100 \mathrm{ml}$ of $2 \mathrm{XYT}$ medium for $4 \mathrm{~h}$ at $30^{\circ} \mathrm{C}$ with shaking. Protein expression was induced by adding $0.1 \mathrm{mM}$ isopropyl $\beta$-D-1-thiogalactopyranoside (IPTG), and the cells were further incubated for $12 \mathrm{~h}$. Cells were harvested by centrifugation, and bacterial pellets were suspended in $10 \mathrm{ml}$ of buffer A (50 mM Tris- $\mathrm{HCl}, 500 \mathrm{mM} \mathrm{NaCl}, \mathrm{pH} 7.8$ ) and disrupted by sonication at $4^{\circ} \mathrm{C}$. Bacterial lysates were centrifuged for $15 \mathrm{~min}$ at $15,000 \times g$ at $4^{\circ} \mathrm{C}$ to obtain cell-free protein extracts. Recombinant proteins were purified from cell-free protein extracts to homogeneity by affinity chromatography according to the TALON ${ }^{\mathrm{TM}}$ purification kit protocol (BD Biosciences) with slight modifications (Chavez-Aviles et al., 2009). Protein concentration was quantified using Bradford reagent (Bio-Rad), and proteins were separated and visualized by $10-12 \%$ sodium dodecyl sulfate-polyacrylamide gel electrophoresis (SDS-PAGE) (Sambrook, 2001). 
TABLE 1 | Strains and plasmids used in this work.

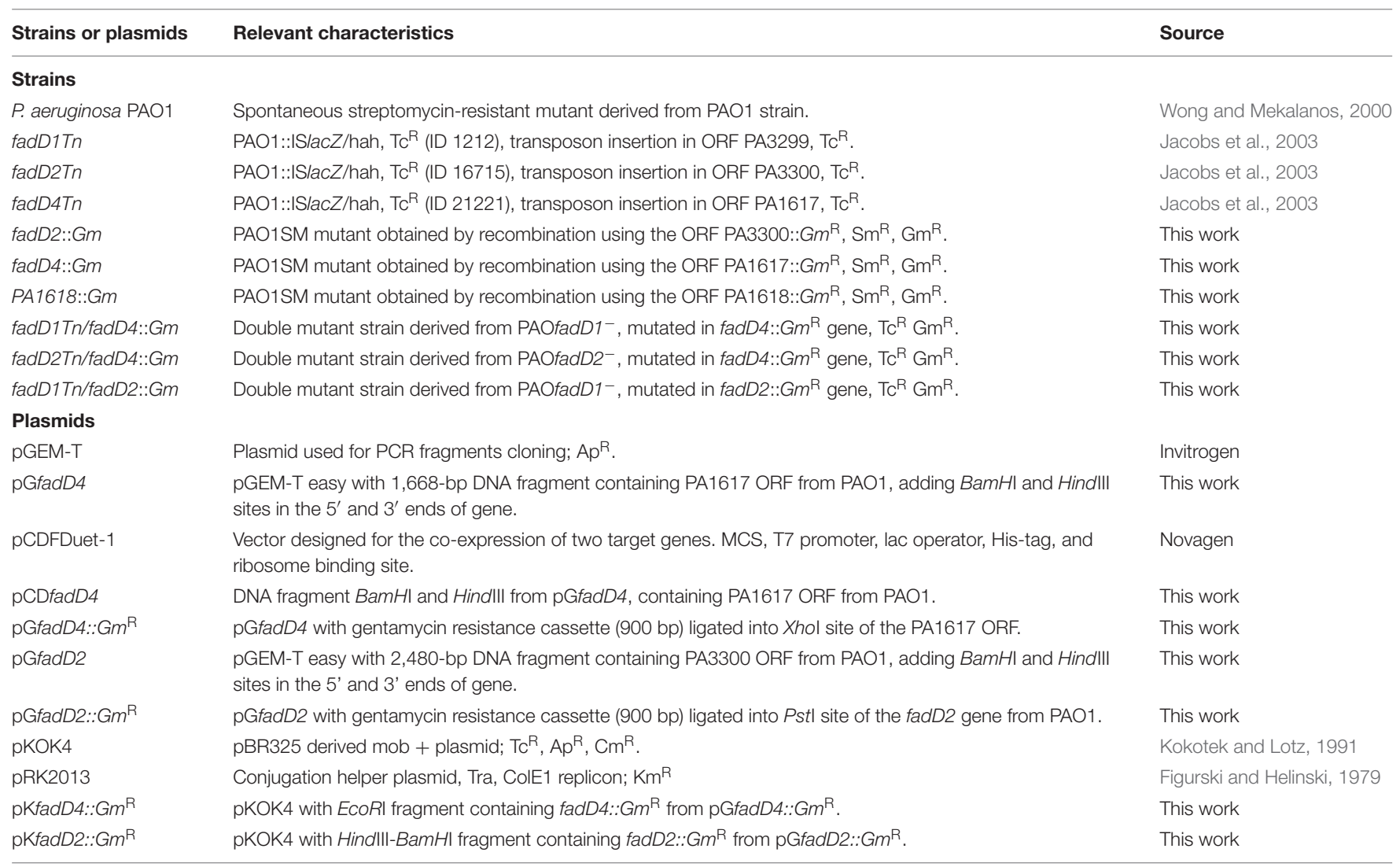

\section{Mutagenesis Procedure}

Construction of mutants (Table 1) was done using triparental conjugation as described (Diaz-Perez et al., 2004), among the $P$. aeruginosa $\mathrm{PAO} 1$ wild-type strain and the single mutants fadD1Tn and fadD2Tn using the pRK2013 helper plasmid (Figurski and Helinski, 1979) and the E. coli S17.1 strain containing the respective plasmids pKGfadD2::Gm ${ }^{\mathrm{R}}$ or pKGfadD4::Gm ${ }^{\mathrm{R}}$ that contain the fadD2 and fadD4 genes disrupted as shown (Table 1). Transconjugants were selected on plates with the appropriate antibiotics and submitted to PCR characterization.

\section{Determination of Acyl-CoA Synthetase Activity}

Acyl-CoA synthetase activity was assayed as described by Hume et al. (2009) with slight modifications. The reaction mixture contained Tris buffer $(50 \mathrm{mM}) \mathrm{pH} 8,12.5 \mu \mathrm{l} \mathrm{MgCl} 2(100 \mathrm{mM})$, $50 \mu \mathrm{l}$ ATP $(100 \mathrm{mM}), 30 \mu \mathrm{l}$ substrate (citronellic, geranic, isovaleric, or fatty acids; $10 \mathrm{mM}$ ), and $100 \mu \mathrm{l}$ of free-cell extract (70 $\mu \mathrm{g}$ protein). The mixture was incubated for $5 \mathrm{~min}$ at $30^{\circ} \mathrm{C}$ in a water bath to equilibration temperature. After, $30 \mu \mathrm{l}$ of coenzyme A (CoA) $(20 \mathrm{mM})$ previously dissolved in Tris buffer $(50 \mathrm{mM}) \mathrm{pH} 8$ was added and incubated for $15 \mathrm{~min}$ at $30^{\circ} \mathrm{C}$ in a water bath. Reactions were stopped by adding $450 \mu \mathrm{l}$ of the ferric chloride reagent, and reaction mixtures were kept on ice for $30 \mathrm{~min}$. The red-purple color absorbance was measured at
$540 \mathrm{~nm}$ in a UV-Vis spectrophotometer. Neutral hydroxylamine solution, $\mathrm{pH}$ 8.0, was prepared by mixing $1 \mathrm{ml}$ hydroxylamine hydrochloride (5 M), $250 \mu \mathrm{l}$ MilliQ water, and $1.25 \mathrm{ml}$ of $\mathrm{KOH}$ (4 M). Ferric chloride reagent was prepared by mixing a 1:1:1 ratio of ferric chloride $(0.37 \mathrm{M})$, trichloroacetic acid $(0.02 \mathrm{M})$, and hydrochloride (0.66 M) (Hume et al., 2009). The molar extinction coefficients of the corresponding fatty acyl hydroxamates were determined experimentally as described (Hume et al., 2009). One unit of enzyme activity is defined as the catalytic activity leading to the formation of $1 \mathrm{nmol}$ of fatty acyl hydroxamate by $1 \mathrm{~min}$. A specific activity is given as unit per milligram of protein.

\section{Extraction and Analysis of Polyhydroxyalkanoates, Rhamnolipid, and Lipopolysaccharides}

For PHA and RHL extraction, P. aeruginosa strains were cultured in phosphate-limited PPGAS medium by $48 \mathrm{~h}$ of incubation at $37^{\circ} \mathrm{C}$. Cells were harvested by centrifugation and washed with $100 \mathrm{mM}$ Tris-100 mM NaCl buffer ( $\mathrm{pH}$ 7). For the PHA extraction, bacterial pellets were disrupted by sonication and digested with $1.8 \%$ sodium hypochlorite for $1 \mathrm{~h}$. After centrifugation, the pellet was washed twice with ethanol and once with acetone (Zhang and Miller, 1992). On the other hand, cellfree supernatants of cultures were used to determine RHL by measuring the rhamnose concentration after acid hydrolysis by the orcinol method (Campos-García et al., 1998). 
The lipopolysaccharide fraction on bacterial pellets obtained by centrifugation of cultures was extracted by using two methods, one as described (Latino et al., 2017) and another by the methanol-chloroform method (Mirzaei et al., 2011). LPS was extracted by chloroform-methanol method; briefly, bacterial pellets were recovered from $15 \mathrm{ml}$ of culture in $\mathrm{LB}$ medium cultured for $48 \mathrm{~h}$ at $37^{\circ} \mathrm{C}$ at $150 \mathrm{rpm}$, washed four times with PBS pH 7.4, resuspended in $2 \mathrm{ml}$ of 95\% ethanol, vortexed for $1 \mathrm{~min}$, and centrifuged at 2,000 rpm for $10 \mathrm{~min}$; the supernatant was discarded and the pellet was washed with 95\% ethanol three times. The pellet was dried and resuspended in $1 \mathrm{ml}$ of $10 \%$ EDTA by sonication. Then, $1 \mathrm{ml}$ of methanol-chloroform (1:2) was added, stirred for $2 \mathrm{~h}$, and centrifuged at 2,000 rpm for $10 \mathrm{~min}$; the upper phase (methanol) was discarded and the lower phase (chloroform) is taken and evaporated for the LPS quantification. LPS extraction samples were run on SDS-PAGE resolving gels (12\%), and gels were stained by silver staining based on standard protocol (Sambrook, 2001; King et al., 2008). Surface polysaccharides of the LPS isolated were measured by the sulfuric acid-phenol colorimetric method using sucrose and fructose as sugar standards (Brimacombe et al., 2013).

\section{Determination of Fatty Acid Content From Polyhydroxyalkanoates and Lipopolysaccharides}

Fatty acid content on PHA and LPS was determined by quantitation of fatty acid methyl esters (FAME) by gas chromatography-mass spectrometry (GC-MS), submitting the samples to methyl esterification as follows. Briefly, total PHA or LPS was dissolved with chloroform-methanol-water and carried out to methyl esterification for $1 \mathrm{~h}$ at $80^{\circ} \mathrm{C}$ in methanol and $\mathrm{H}_{2} \mathrm{SO}_{4}$ (Campos-García et al., 2018). The FAME were extracted with hexane, samples were suspended in $500 \mu \mathrm{l}$ of chloroform, and $1 \mu \mathrm{l}$ was analyzed using GC-MS (GC; Agilent 6850 Series II equipped with MS-5973 and FID detectors), using a Zebron ZB-FFAP column, 30-m length, I.D. $0.25 \mathrm{~mm}$, and film $0.25 \mathrm{~mm}$ (Phenomenex) (Campos-García et al., 2018). Sample injection was performed at a splitless mode at a temperature of $280^{\circ} \mathrm{C}$ and an FID detector temperature of $300^{\circ} \mathrm{C}$. The oven temperature was programmed to start at $50^{\circ} \mathrm{C}$, maintained in isothermal for $5 \mathrm{~min}$, then increased to $280^{\circ} \mathrm{C}$ at a rate of $10^{\circ} \mathrm{C} / \mathrm{min}$, and then isothermal $280^{\circ} \mathrm{C}$ for $5 \mathrm{~min}$. Compounds were identified by comparison with the mass spectral library (NIST/EPA/NIH, ChemStation, Agilent Technologies Rev. D.04.00 2014). FAME mass profiles were confirmed by comparing with FAME Mix $\mathrm{C}_{4-} \mathrm{C}_{24}$ standard (Supelco). Quantitation was performed using the relative values of the peak areas in chromatograms obtained by using an FID detector. Results correspond to means of triplicate assays of PHA and LPS samples.

\section{Determination of Other Virulence Factors and Survival of Caenorhabditis elegans}

Pyocyanin and quorum sensing (QS) AHL-dependent assays were determined in the cell-free supernatant fraction of $P$. aeruginosa cultures grown in $\mathrm{LB}$ medium at $37^{\circ} \mathrm{C}$ for 24 and $48 \mathrm{~h}$. Pyocyanin was extracted from $1 \mathrm{ml}$ of the cellfree supernatant mixed with $1 \mathrm{ml}$ of chloroform, and the organic fraction was collected and mixed with $1 \mathrm{ml} 0.2 \mathrm{~N}$ $\mathrm{HCl}$; subsequently, the organic fraction was separated, and the absorbance was measured at $520 \mathrm{~nm}$ in a spectrophotometer (Gonzalez et al., 2017). The QS AHL-dependent assay was performed using the E. coli JM109 reporter strain transformed in the pSB1075 plasmid, which contains the transcriptional fusion plasRlasIRhlI:luxCDABE and produces luminescence in response to 3-oxo-C 12 -HSL (Winson et al., 1998). Briefly, the E. coli JM109 (pSB1075) strain was grown overnight in LB liquid medium at $37^{\circ} \mathrm{C}$ with shaking. Then, the culture was diluted with fresh LB medium to obtain an optical density (OD) of 0.1 at $600 \mathrm{~nm}$ $\left(\mathrm{OD}_{600}\right)$, and $200 \mu \mathrm{l}$ was distributed in each well of a 96 -well culture plate and incubated for $2 \mathrm{~h}$ at $37^{\circ} \mathrm{C}$. Subsequently, $50 \mu \mathrm{l}$ of cell-free $P$. aeruginosa culture supernatants were added, followed by incubation at $37^{\circ} \mathrm{C}$ with slight shaking. Both the $\mathrm{OD}_{600}$ and luminescence were determined at $4 \mathrm{~h}$ using a ChemiDoc MP imaging system (Bio-Rad) (Gonzalez et al., 2017).

For quantification of biofilm, cultures of $P$. aeruginosa strains were adjusted to $\mathrm{OD}_{600}=0.1$ optical density with LB medium, and 100- $\mu \mathrm{l}$ aliquots of diluted cultures were collocated into 96-well microtiter plates and incubated at $37^{\circ} \mathrm{C}$ for $48 \mathrm{~h}$. Culture media were eliminated, and biofilm quantification was done by dyeing the 96-well plate with crystal violet and measuring the absorbance of samples in the plate at $550 \mathrm{~nm}$ (Coffey and Anderson, 2014).

Survival assays using C. elegans Bristol N2 and CL2166 dvIs19 [pAF15 (Pgst-4::GFP::NLS)] worms (provided by the Caenorhabditis Genetics Center, University of Minnesota) were synchronized by hypochlorite isolation of eggs from gravid adults (Stiernagle, 2006). Briefly, L1 larvae were transferred onto nematode growth medium plates seeded with the E. coli OP50 strain previously grown on the plates as a food source and were incubated at $18^{\circ} \mathrm{C}$ for $4-5$ days until they reached the young adult phase. Worms were incubated either with cell-free medium collected from bacterial cultures grown for 24 and $48 \mathrm{~h}$ in LB medium (slow-killing assay) or bacterial strains (fast-killing assay). For each experiment, 20-30 worms were placed, and plates were incubated at $25^{\circ} \mathrm{C}$ and scored for life at $6,12,24$, and $48 \mathrm{~h}$. For statistical purposes, at least three replicates per trial were carried out. E. coli OP50 culture supernatant was used as a negative control. C. elegans worms were considered dead when they stopped moving and did not respond to a nudge with a platinum wire. The fluorescence microscopy images obtained were taken using a fluorescent and phase contrast inverted microscope (Nikon Eclipse TE300) with Plan Fluor $4 \times$ and $10 \times$ dry lenses, and an AmScope ML300 3.1-MP digital color camera.

\section{Western Blot Analysis}

Cell-free protein extracts were used for immunoblot assays. Thirty micrograms of total protein previously quantified was mixed with $10 \mu \mathrm{l}$ of denaturing buffer (Tris- $\mathrm{HCl} 0.06 \mathrm{M}, \mathrm{pH}$ 6.8, 5\% glycerol, 4\% SDS, $4 \% \beta$-mercaptoethanol, and $0.0025 \%$ bromophenol blue) for $5 \mathrm{~min}$ at $95^{\circ} \mathrm{C}$ in a boiling water bath. Samples were run in SDS-PAGE at $10 \%$. The gels in one side were Coomassie blue stained and the other gels were transferred 
to polyvinylidene difluoride (PVDF, Millipore) membrane. For immunodetection, membranes were blocked using dry milk in TBS-T (Tris-HCL $10 \mathrm{mM}$; $\mathrm{NaCl}$ 0.9\%; and 0.1\% Tween-20, $\mathrm{pH}$ 7.8) and blotted with the respective antibody. The FadD4 protein was detected using anti-His-tag antibody (Invitrogen) and the anti-mouse-HRP conjugate as the secondary antibody (Santa Cruz Biotechnology) as indicated by the provider. His-tag SDS-PAGE protein standard (Invitrogen) was used as molecular markers. On the other hand, the antibodies IL-8 sc-376750, IL-1 $\beta$ sc-32294, and IL-1 $\beta$ sc-52012 (Santa Cruz Biotechnology) were blotted in a blocking medium at 1:5,000 dilution overnight at $4^{\circ} \mathrm{C}$ with light shaking. After washing, membrane was incubated with the secondary antibody, Goat anti-Mouse IgG HRP conjugate (Bio-Rad), in a blocking medium at 1:10,000 dilution for $2 \mathrm{~h}$ at $4^{\circ} \mathrm{C}$. Membranes were washed twice with TBS-T and developed using hydrogen peroxide and SuperSignal West Pico Luminol, exposing in ChemiDoc ${ }^{\mathrm{TM}}$ MP System (Bio-Rad). At least three independent assays were conducted, and representative images are shown. Band intensities in images were quantified using the ImageJ1 software (NIH Image).

\section{Rat Exposition to Lipopolysaccharides}

Twenty four healthy Wistar rats ( $\geq 250 \mathrm{~g}$ body weight) were housed in rodent plastic boxes and placed in a temperature/humidity/light-controlled chamber set at $22 \pm 1^{\circ} \mathrm{C}$, 12:12-h light/dark cycle, and unlimited access to food and water before treatment. Animal handling, feeding, and care were done by trained personnel following the National Institutes of Health Guide for the care and use of laboratory animals. This research was also approved by the Institutional Committee for Use of Animals of the I.I.Q.B/Universidad Michoacana de San Nicolás de Hidalgo in agreement with NOM-062-ZOO1999, Ministry of Agriculture, Mexico. Lipopolysaccharides extracted from $P$. aeruginosa PAO1 strains were dissolved in sterile $0.9 \%$ sodium chloride and injected intraperitoneally at a dose of $50 \mu \mathrm{g} / \mathrm{kg}$ (Kozak et al., 2006). Body temperature was monitored every $15 \mathrm{~min}$ for $4 \mathrm{~h}$, and finally, the animals were euthanized by receiving an overdose of sodium pentobarbital. Blood was collected, white blood cell count was examined by microscopic observation, and serum was separated for interleukin determination by western blot.

\section{Statistical Analysis}

For correlation data analysis, data obtained in all assays were analyzed by utilizing response variables (strains) vs. numerical data for each assay (cases) using the STATISTICA software (Data Analysis Software System 8.0; Stat Soft Inc.). Other data were statistically analyzed using GraphPad Prism 6.0 software (GraphPad Software Inc.).

\section{RESULTS AND DISCUSSION}

\section{Genomic Context of the fadD4 Gene in $P$. aeruginosa PAO1}

Multiple homologs of fadD genes have been identified in $P$. aeruginosa PAO1, which are involved in FA catabolism and are also related to virulence; however, its genetic redundancy is uncertain in terms of bacterial pathogenicity (Kang et al., 2010; Zarzycki-Siek et al., 2013). In this study, an exhaustive bioinformatics analysis indicated that the PAO1 genome contains approximately 30 proteins paralogous to FadD1/FadD2 (proteins with 500-700 amino acids showing at least the AMP-binding protein motif) (Supplementary Table 1). The ORF PA1617 in the $P$. aeruginosa PAO1 genome, which was designated as fadD4 gene in an earlier report (ZarzyckiSiek et al., 2013), encodes a protein of 555 residues with a predicted molecular weight of $61.4 \mathrm{kDa}$ (Pseudomonas Genome Database, PGD). The PA1617-encoded protein contains the characteristic domains for ATP/AMP binding found in the fatty acyl-AMP ligase/fatty acyl-CoA synthetases (YTSGTTGVPKGA-N117-EVYGMTE) and the fatty acidbinding domain (DGFLRTGDKGEQDADGNLRLTGRMR), which indicates that it belongs to the family of fatty acyl-CoA synthetases (FadDs) involved in fatty acid degradation (Figure 1).

Pseudomonas aeruginosa PAO1 contains multiple homologous proteins that show both the characteristic ATP/AMP binding and fatty acyl-CoA domains, suggesting that they could be involved in AMP activation of multiple substrates and participate in multiple metabolic pathways, in addition to FA oxidation (Zarzycki-Siek et al., 2013). In this context, our BLAST protein sequence analysis showed 12 highly conserved paralogous proteins, containing the FadD protein domains. The PA1617 ORF from $P$. aeruginosa shared an amino acid sequence identity of $24.6 \%$ with FadD1 (PA3299), 25.2\% with FadD2 (PA3300), 27.8\% with FadD3 (PA3860), 22.5\% with FadD5 (PA2893, also called AtuH), and 24.9\% with FadD6 (PA3924). In addition, it shared a similar sequence identity with the remaining paralogs: 23\% with PA0887, 27.2\% with PA0996, 24.8\% with PA2557, 24.4\% with PA3568, 24\% with PA4198, 25.6\% with PA4228, and 22.1\% with PA4733 (Figure 1). Interestingly, FadD4 from $P$. aeruginosa showed good sequence identity with homologous proteins from other bacterial species, such as the feruloyl-CoA synthetase (Fcs) from Pseudomonas fluorescens BF13 (Calisti et al., 2008) (25.6\% identity) and the isoprenoyl-CoA synthetases Acs1 and Acs2 from Marinobacter hydrocarbonoclasticus DSM8798 strain (24.1 and 25.2\%, respectively) (Holtzapple and Schmidt-Dannert, 2007). However, the highest identities and nearest phylogenetic relationships of the PA1617 ORF were observed with the Acs3 and Acs4 proteins from M. hydrocarbonoclasticus DSM8798, showing 55.4 and $49.5 \%$ identity, respectively, and with the Fsc protein from $P$. fluorescens BF13 (Figures 1, 2A). It has been reported that $M$. hydrocarbonoclasticus DSM8798 contains several AMP-forming long-chain acyl-CoA synthetases (Acs1-Acs4), which are involved in the CoA activation of fatty acids and isoprenoid fatty acids required for isoprenoid wax ester synthesis when the bacterium is grown on phytol (Holtzapple and Schmidt-Dannert, 2007).

In the resulting phylogenetic tree, four clusters can be clearly observed: FadD1, FadD2, and FadD3 are grouped in the same cluster with the paralogous ORF PA4228 and the isoprenoyl-CoA synthetase (Asc2) from M. hydrocarbonoclasticus DSM8798, but FadD5 and FadD6 are located outside the cluster (Figure 2A). 


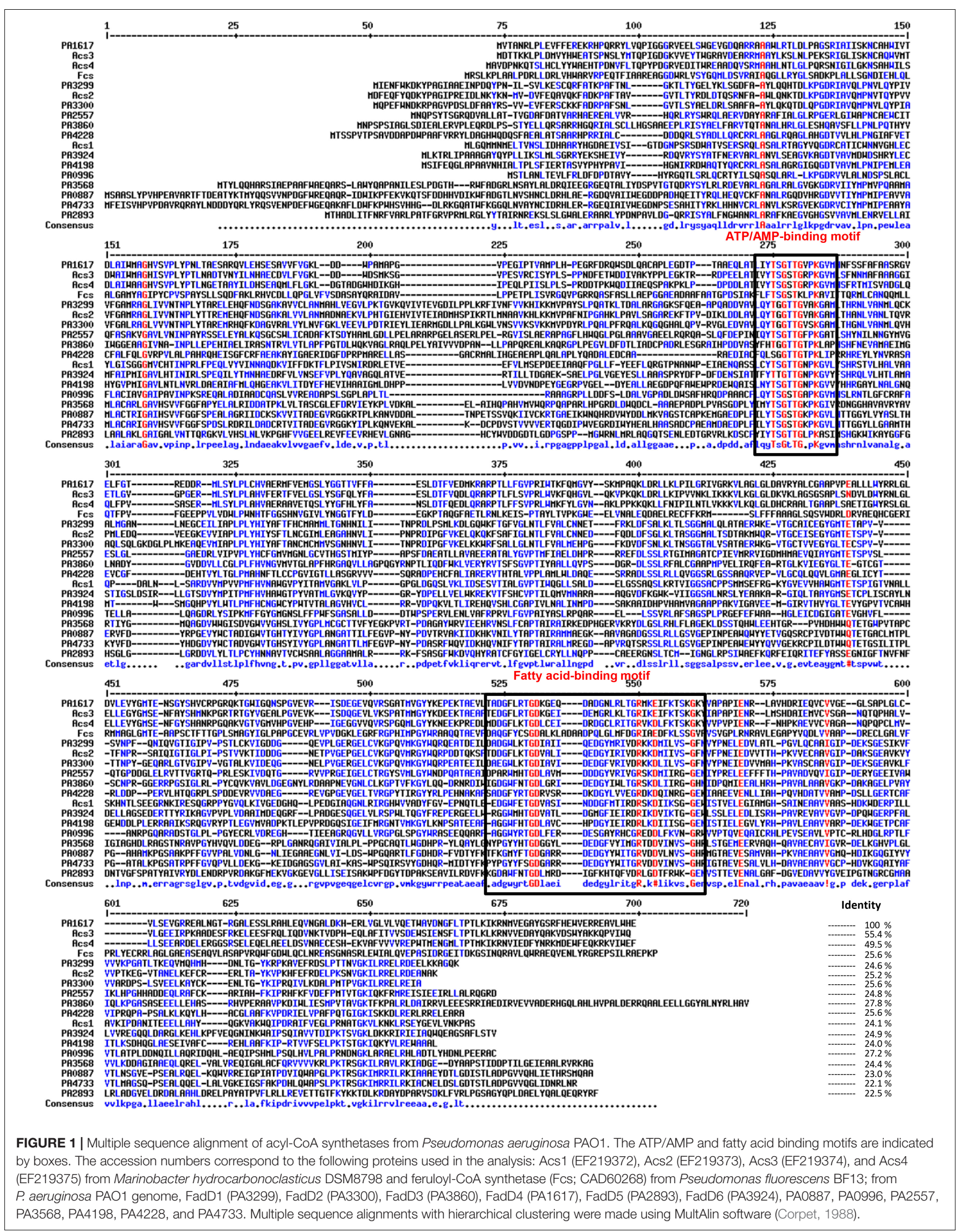




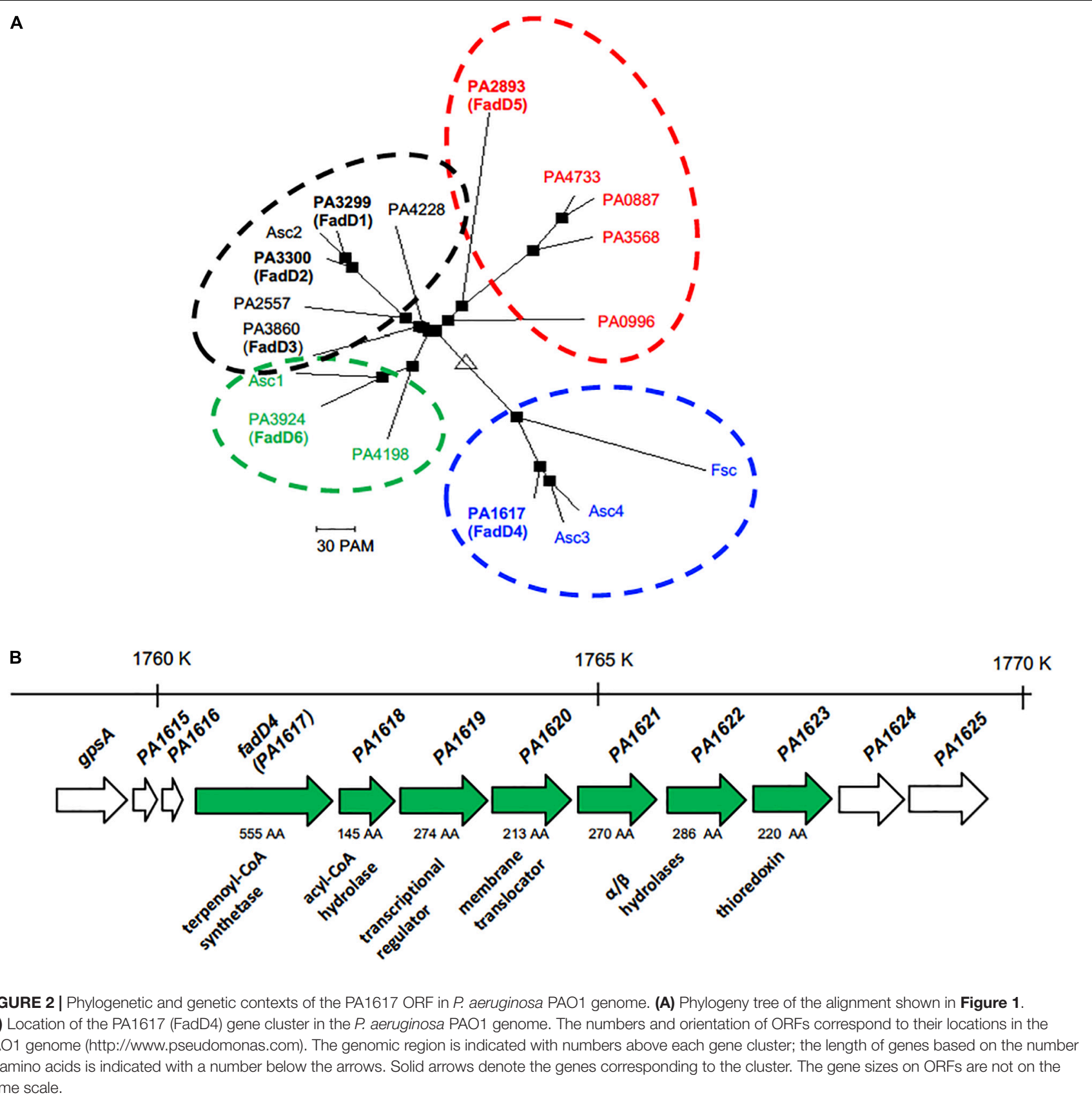

FadD5 was grouped with the PA0887, PA0996, PA3568, and PA4733 ORFs, while FadD6 clustered with the PA4198 ORF and the Acs1 protein from M. hydrocarbonoclasticus DSM8798. Interestingly, PA1617 (FadD4) was grouped external to others with the Asc3 and Asc4 proteins from M. hydrocarbonoclasticus DSM8798 and feruloyl-CoA synthetase (Fcs) from P. fluorescens BF13 (Figure 2A). These data allowed us to hypothesize that the ORF PA1617 from $P$. aeruginosa PAO1, named fadD4 gene, could encode the main functional terpenoyl-CoA synthetase from $P$. aeruginosa. Interestingly, bioinformatics findings indicated a distant phylogenetic relationship of the FadD4 with the FadD1/D2/D3/D5/D6 paralogs, as codon usage comparison between fadD4 (PA1617) and fadD1 genes also showed significant differences in bias and frequency of rare used codons (Supplementary Figure 1), suggesting that the fadD4 gene might have been acquired by horizontal transfer from other bacterial species and, consequently, could exhibit a different biological function.

In addition, our computational analysis suggests that a possible operon may be constituted by the ORFs PA1617 to PA1623 (Figure 2B). The putative function of PA1618 is related to the 4-hydroxybenzoyl-CoA thioesterase enzyme, which could play roles in thioester hydrolysis in fatty acid metabolism. The following PA1619 and PA1620 ORFs share a sequence 
TABLE 2 | Growth analysis of $P$. aeruginosa strains in different compounds.

\begin{tabular}{|c|c|c|c|c|c|c|c|}
\hline \multirow[t]{2}{*}{ Strain } & \multicolumn{7}{|c|}{ Compound } \\
\hline & Octanol & Citronellol & Geraniol & Citronellic & Geranic & Leucine & Isovaleric \\
\hline PAO1 (WT) & +++ & +++ & ++ & +++ & +++ & +++ & +++ \\
\hline fadD1Tn & +++ & ++ & + & ++ & ++ & +++ & +++ \\
\hline fadD2Tn & +++ & ++ & + & ++ & ++ & +++ & +++ \\
\hline fadD1Tn/fadD2::Gm & +++ & ++ & ++ & +++ & ++ & +++ & + \\
\hline fadD4Tn & +++ & ++ & ++ & ++ & ++ & +++ & +++ \\
\hline fadD4::Gm & +++ & + & $-(+/-)$ & + & + & +++ & ++ \\
\hline PA1618::Gm & +++ & + & $-(+/-)$ & + & + & +++ & ++ \\
\hline fadD1Tn/fadD4::Gm & +++ & $-(+/-)$ & $-(+/-)$ & $-(+/-)$ & $-(+/-)$ & +++ & +++ \\
\hline fadD2Tn/fadD4::Gm & +++ & $-(+/-)$ & $-(+/-)$ & $-(+/-)$ & $-(+/-)$ & +++ & ++ \\
\hline
\end{tabular}

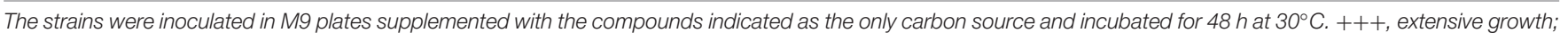

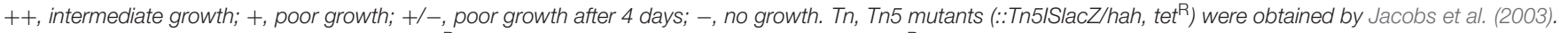
Single and double mutants in fadD genes $\left(: \because \mathrm{Gm}^{\mathrm{R}}\right)$ were obtained by gene disruption using the $\mathrm{Gm}^{\mathrm{R}}$ cassette by homolog recombination.

similarity with transcriptional regulators of the AraC family and transmembrane solute translocators, respectively. PA1621 and PA1622 are homologs of the alpha/beta hydrolase family, such as pimeloyl-ACP methyl ester carboxylesterase, and finally, the PA1623 ORF is homologous to the thioredoxin-like superfamily.

\section{The fadD4 Gene Is Implicated in Acyclic Terpene and Fatty Acid Assimilation}

The functions of the paralogs fadD1-D6 in the P. aeruginosa PAO1strain have been described to encode enzymes for fatty acyl-CoA activation, recognizing FA substrates of different chain lengths; however, its genetic redundancy, bacterial metabolism, and virulence are not clearly known yet. The fadD1 and fadD2 genes in the $P$. aeruginosa PAO1 strain have been described to encode acyl-CoA synthetases that recognize FA substrates of different chain lengths and are also related with phosphatidylcholine assimilation (Zarzycki-Siek et al., 2013). However, the functions of the paralogs FadD3-FadD6 are not clearly known yet. A mutagenesis approach and a functional study let us delve deep in elucidating these implications. Previous mutagenesis studies in our laboratory on the P. aeruginosa PAO1 strain showed that the ORF PA1617 mutation impairs the ability to grow on acyclic terpenes such as citronellol (Campos-García, 2010). Therefore, the ability of some of the fadD mutants to grow on acyclic terpenes was evaluated.

The fadD1Tn and fadD2Tn mutants, which were obtained by transposon insertion into the fadD1 (ORF PA3299) and fadD2 (ORF PA3300), respectively (Table 1), were capable of growing on solid minimal medium supplemented with (as sole carbon source) $n$-octanol, leucine, or isovaleric acid, similar to the wild-type (WT) strain, but their growth was impaired on terpenes such as citronellol, geraniol, citronellic acid, or geranic acid (Table 2). In agreement, the transposon-obtained mutant fadD4Tn (transposon mutant in ORF PA1617) showed a similar ability to grow on terpenes as fadD1 and fadD2 mutants (Table 2). Interestingly, a mutant obtained by genetic recombination of a disrupting antibiotic cassette into the ORF PA1617 (fadD4::Gm) showed a clear impairment of growth on acyclic terpenes (Table 2). This result indicates that both the fadD1 and fadD2 genes may be involved in acyclic terpene assimilation, being that both genes are not essential, probably due to genetic redundancy. This observation is in agreement with the ability of FadD homologs to activate acyclic terpenes of a wide range of chain lengths, suggesting that the $P$. aeruginosa FadD1 and FadD2 enzymes could also activate acyclic terpenoic acids (Kang et al., 2010; Zarzycki-Siek et al., 2013).

To verify this assumption, a double mutant of the fadD1 and fadD2 genes was constructed. The transposon-obtained mutant fadD1Tn was mutagenized with the fadD2 gene, utilizing the fadD2 PCR product obtained by disruption with a cassette of gentamicin resistance $(f a d D 2:: G m)$ (Table 1). Interestingly, the double mutant fadD1Tn/fadD2::Gm was capable of growing on acyclic terpenes, similar to their single mutants (Table 2). This result not only confirms that, in the $P$. aeruginosa PAO1 strain, the fadD1 and fadD2 genes are not essential for acyclic terpene assimilation but also confirms the existence of homolog genes that encode to a terpenoyl-CoA synthetase.

The participation of fadD4 in acyclic terpene catabolism and its genetic redundancy were elucidated by the construction of double mutants in combination with fadD1 and fadD2 mutants, by utilizing the disrupted PA1617 ORF with a gentamicin resistance cassette $($ fadD4::Gm) (Table 1). The fadD1Tn/fadD4::Gm and fadD2Tn/fadD4::Gm double mutants were unable to grow on acyclic terpenes (citronellol, geraniol, citronellic acid, and geranic acid); however, these mutants were capable to grow on leucine and isovaleric acid (Table 2). In contrast, as mentioned above, the fadD1Tn/fadD2::Gm double mutant grew on the terpenes tested.

Additionally, the disrupted fadD4::Gm single mutant and the double mutants fadD1Tn/fadD4::Gm and fadD2Tn/fadD4::Gm were unable to grow efficiently on liquid M9 medium with citronellic acid as the sole carbon source, whereas the double mutant fadD1Tn/fadD2::Gm and the single mutants fadD1Tn and fadD2Tn did not show any significant difference in growth compared to the WT strain (Figure 3A). Although the growth on isovaleric acid was slightly affected in some mutants, they were capable of growing as well as the WT strain, with palm 

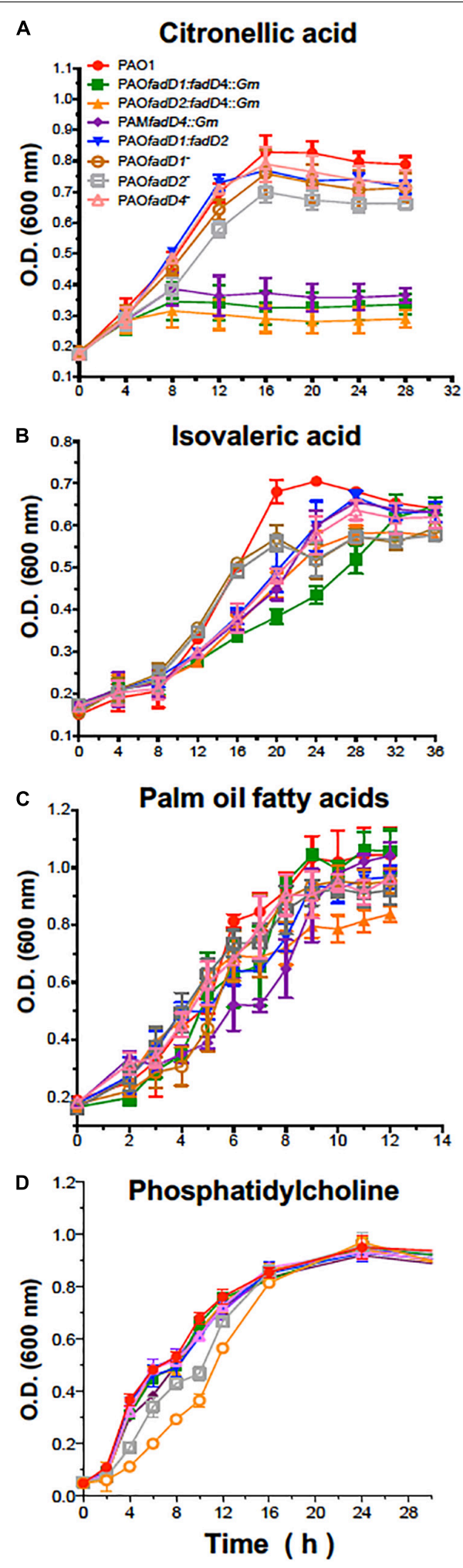

FIGURE 3 | Bacterial growth of $P$. aeruginosa PAO1-derived strains. Cultures were grown on $\mathrm{M} 9$ minimal medium with $0.1 \%$ citronellic acid $\mathbf{( A )}, 0.1 \%$ isovaleric acid (B), $0.1 \%$ oil fatty acids (C), or $0.4 \%$ phosphatidylcholine (D) at $30^{\circ} \mathrm{C}$ with shaking. Results are shown as means $\pm \mathrm{SE}$ of triplicate experiments. oil FA and phosphatidylcholine as carbon sources (Figures 3BD). These results indicate that in $P$. aeruginosa PAO1, the fadD4 gene is essential for the assimilation of acyclic terpene, but not for leucine/isovalerate, suggesting that fadD4 encodes for the terpenoyl-CoA synthetase, further confirming the existence of genetic redundancy in the $f a d D$ genes.

In this study, the mutant $f a d D 4 T n$, whose transposon is located in the first third of the PA1617 ORF, was deficient but not incapable to grow on acyclic terpenes. In contrast, the growth of PA1618::Gm mutant obtained by disruption of the PA1618 ORF was severely impaired on terpenes as sole carbon sources (Table 2). These growth phenotypes may be explained by the fadD4Tn mutant not causing polar effects over downstream genes contained in the putative operon identified (Figure 2), allowing the genetic expression of the downstream genes; in contrast, the gene disruption in the fadD4::Gm and PA1618::Gm mutants prevents the expression of downstream genes due to the presence of a transcription terminator sequence in the resistance cassette. These effects suggest that the downstream genes (ORF PA1618 to PA1623) could be involved in subsequent steps of acyclic terpene and fatty acid degradation; in addition, mutations in the fadD 4 and PA1618 ORFs by gene disruption reveal that the fadD 4 operon plays an essential role in acyclic terpene assimilation, suggesting that other ORFs that constitute the operon could also be involved; however, further investigation is required to elucidate their function.

\section{Enzymatic Activity of the Recombinant FadD4 Protein}

To study the function of the FadD4 protein, its expression level and enzymatic activity were determined. The fadD4 gene (PA1617) was amplified by PCR as a 1,668-bp DNA fragment, and the $f a d D 1$ and $f a d D 2$ genes were also subcloned separately into an expression vector designed to merge a 6-histidine tag into the N-terminal ends of the FadD proteins and introduced by electroporation into E. coli JM101, as described in Section "Materials and Methods." Cell-free protein extracts of cultures induced with IPTG were monitored for recombinant protein expression on SDS-PAGE gels. After purification by affinity chromatography, we observed a $\sim 70-\mathrm{kDa}$ protein for the $f a d D 1$ gene product, two bands of $\sim 63-68 \mathrm{kDa}$ for the $f a d D 2$ gene product, and a $\sim 62-\mathrm{kDa}$ protein band corresponding to the fadD4 gene product, all of which were confirmed by western blot using anti-His antibody (Figure 4). Additionally, the FadD4 and FadD1 proteins were located in the soluble fraction, while the recombinant FadD2 protein was mainly located in the membrane fraction (Figure 4). These data suggest that both FadD1 and FadD4 could be cytoplasmic or extracellular proteins, while the FadD2 protein could be associated with the membrane.

The recombinant FadD4 protein was purified by affinity chromatography, and the dialyzed fraction was used for enzymatic activity determination. Using this procedure, we were unable to isolate a stable fraction with activity for subsequent enzyme characterization; therefore, cell-free protein extracts of cultures of E. coli BL21(DE3) transformed with respective 


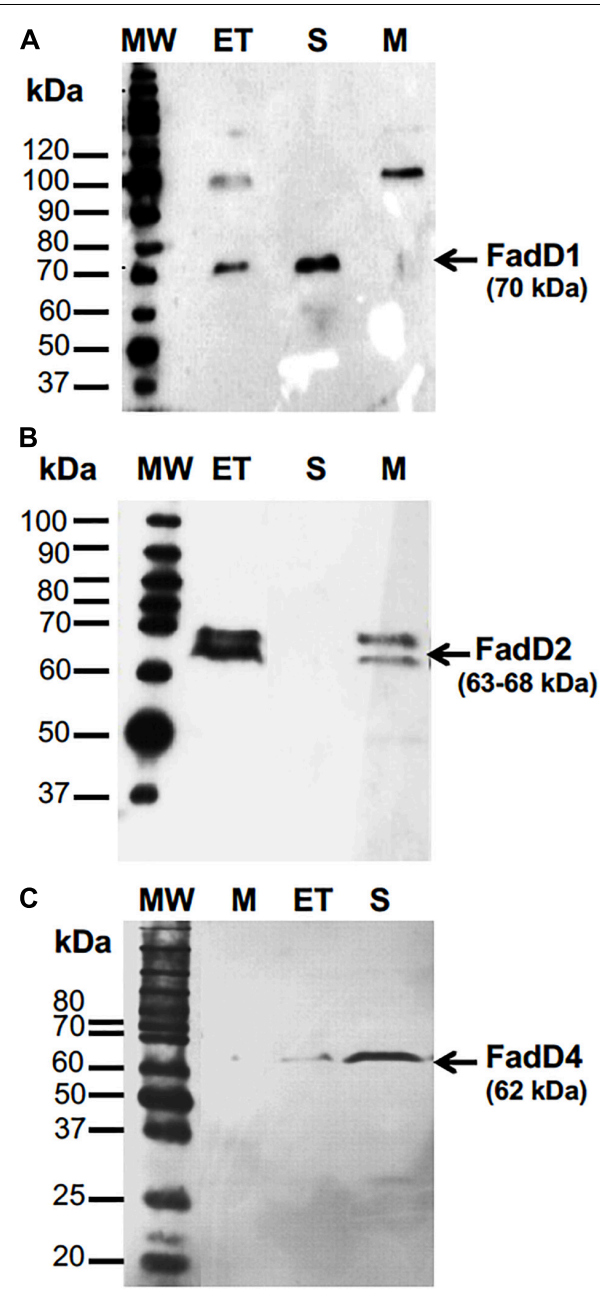

FIGURE 4 | The expression of recombinant FadD1, FAdD2, and FadD4 proteins of $P$. aeruginosa PAO1 in E. coli. Purification and western blot analysis of (A) FadD1, (B) FadD2, and (C) FadD4. Western blot detection used anti-His antibody. MW, molecular mass marker; ET, cell-free protein extract; S, soluble fraction; M, membrane fraction. Protein molecular weight markers in kilodaltons are indicated.

plasmids were used to determine the acyl-CoA synthetase activity following a previously described method (Kang et al., 2010).

Protein extracts of $E$. coli overexpressing the recombinant FadD4 protein showed similar levels of enzymatic activity with different substrates, such as citronellic acid $(418 \mu \mathrm{mol} / \mathrm{min} / \mathrm{mg})$, geranic acid (336 $\mu \mathrm{mol} / \mathrm{min} / \mathrm{mg}$ ), and long-chain fatty acids $\left(\mathrm{C}_{12}-\mathrm{C}_{18} \mathrm{mix} ; 358 \mu \mathrm{mol} / \mathrm{min} / \mathrm{mg}\right)$, and reduced activity with isovaleric acid (148 $\mu \mathrm{mol} / \mathrm{min} / \mathrm{mg}$ ) (Table 3 ). These results indicate that the FadD4 protein in $P$. aeruginosa PAO1 encodes an acyl-CoA synthetase capable of using acyclic terpenoic acids, fatty acids, and isovaleric acid as substrates. This finding indicates that, like FadD1 and FadD2, FadD4 is active on a wide range of substrates. However, the growth phenotype indicates that FadD4 corresponds to the terpenoyl-CoA synthetase, which is preferentially required for acyclic terpene assimilation, suggesting that FadD1/D2 versus FadD4 is subject to different
TABLE 3 | Acyl-CoA synthetase activity in cell-free extracts of E. coli expressing the fadD4 gene from $P$. aeruginosa PAO1.

\begin{tabular}{lcc}
\hline Substrate & \multicolumn{2}{c}{ Acyl-CoA synthetase $(\mu$ moles/min $\times$ mg protein) } \\
\cline { 2 - 3 } & E. coli & E. coli (pCDfadD4) \\
\hline Citronellic acid & $30 \pm 8$ & $418 \pm 132^{\star}$ \\
Geranic acid & $0 \pm 4$ & $336 \pm 57^{\star}$ \\
Isovaleric acid & $8 \pm 4$ & $148 \pm 50$ \\
Long-chain fatty acids & $25 \pm 5$ & $358 \pm 92^{\star}$ \\
\hline
\end{tabular}

Recombinant FadD4 enzyme was expressed in Escherichia coli BL21(DE3) strain, and cell-free extracts were used for determination of acyl-CoA synthetase specific activity. The activity was determined using $10 \mathrm{mM}$ of substrates, $10 \mathrm{mM}$ ATP, $10 \mathrm{mM} \mathrm{Mg}{ }^{2+}$ in phosphate buffer, $\mathrm{pH} 7.0$, and $30^{\circ} \mathrm{C}$ by 30 -min reaction (Kang et al., 2010). Values of means $\pm S D$ were analyzed by multiple $t$-test-one; significant differences with respect to basal activity are indicated $\left(^{*}\right), n=3, p<0.05$.

genetic regulation processes depending on the substrate and growth conditions.

\section{The fadD4 Mutation Modifies the Production of Virulence Factors}

FadD paralog proteins (FadD1 to FadD6) have been described to be involved in the degree of pathogenesis and virulence mechanism of $P$. aeruginosa PAO1. Regarding the FadD1 and FadD2 members of the FA regulon, FadD2 mutants exhibited a decreased production of lipase, protease, rhamnolipids, and phospholipase; both mutants showed noticeable deficiencies in assimilating FAs and phosphatidylcholine, which translated to a decreased infection capability in a mouse lung model (Kang et al., 2010). FadD1 plays a dominant role in FA metabolism, whereas FadD2 is activated only when FadD1 is inactivated, suggesting different levels of regulation. High genetic redundancy in $P$. aeruginosa increases the uncertainty about the function of FadD enzymes. However, a link between FA degradation, nutrient metabolism, and the expression of virulence factors is known. FadD1 and FadD2 mutants showed decreased competition levels in lung colonization, with the FadD2 mutant and the double mutant FadD1D2 being more deficient than the FadD1 mutants (Kang et al., 2010); however, the authors described that a significant modification in the production of virulence factors (such as protease, hemolysin, lipases, phospholipases, and rhamnolipids) was not observed, except in the multiplemutant fadD1D2D3D4D5D6, which showed diminished levels (Kang et al., 2010; Zarzycki-Siek et al., 2013). As the multiple mutagenesis procedure may be associated with pleiotropic effects, we considered to re-evaluate the FadDs' roles in the pathogenesis of $P$. aeruginosa; therefore, a construction of novel mutants in the fadD1, fadD2, and fadD4 genes by means of a different methodology was carried out to elucidate whether they are involved.

The las/rhl systems of QS have been described to hierarchically control the production of major virulence factors in $P$. aeruginosa (Lee and Zhang, 2015). Thus, we evaluated the ability of the culture supernatants of the $P$. aeruginosa strains (PAO1 WT, fadD1Tn, fadD2Tn, fadD4::Gm, fadD1Tn/fadD2::Gm, fadD1Tn/fadD4::Gm, and fadD2Tn/fadD4::Gm) to induce 


\section{A}
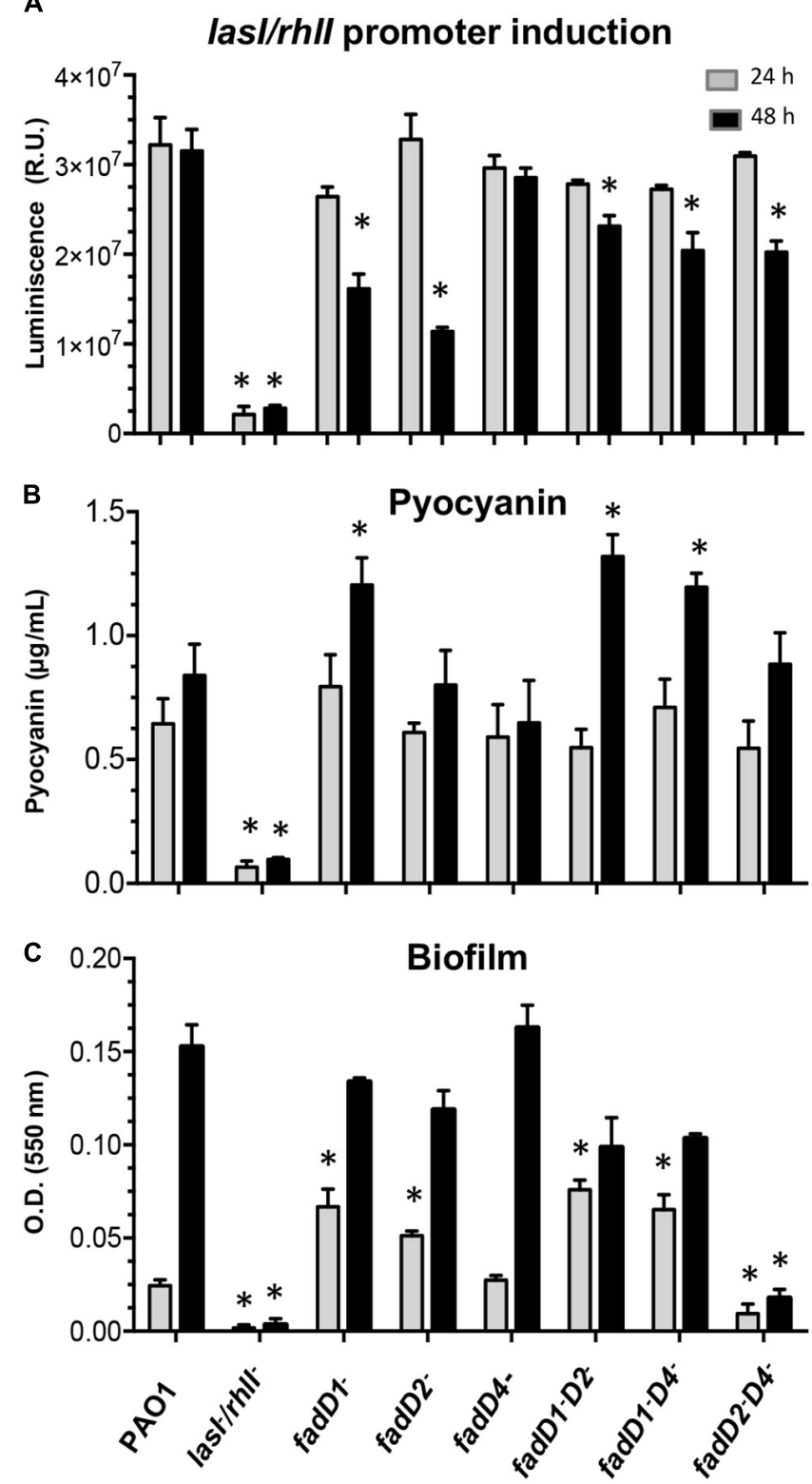

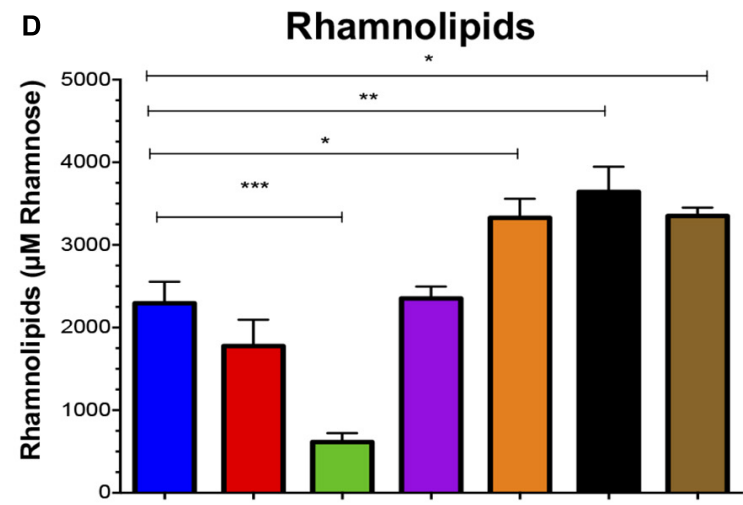

E Poly-hydroxyalkanoates

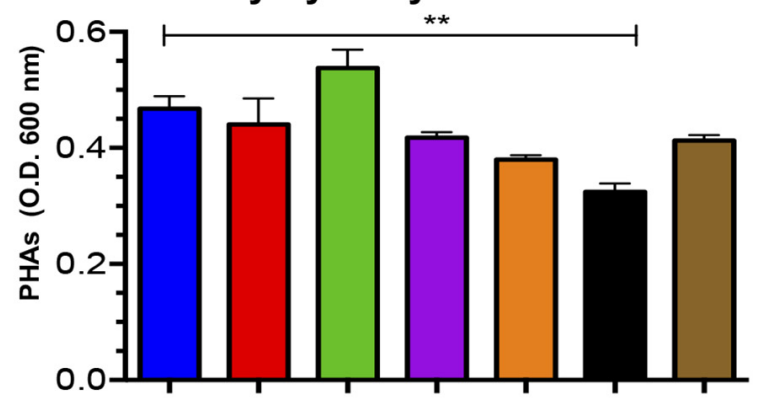

$\mathbf{F}$

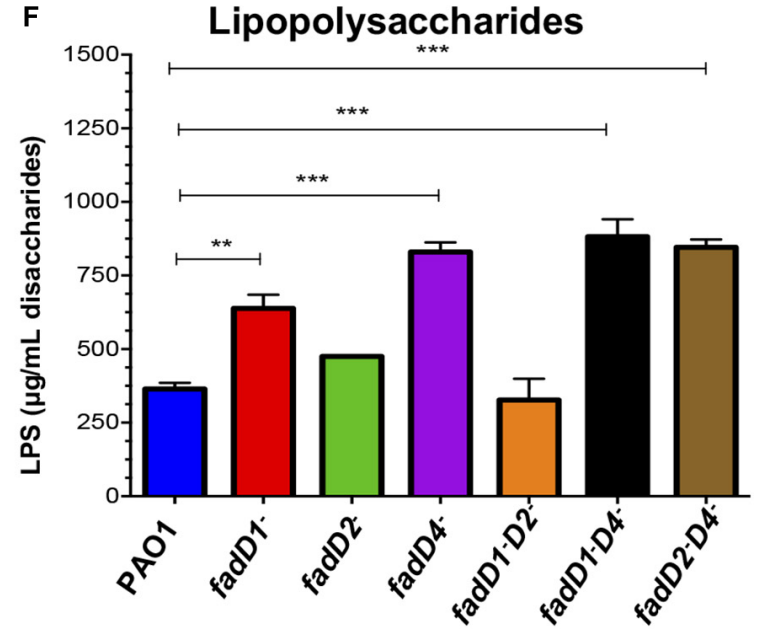

FIGURE 5 | Determination of virulence factors in the cultures of $P$. aeruginosa PAO1 fadD mutants. (A-C) Determination of virulence factors in the cell-free supernatants of $P$. aeruginosa PAO1 strains as described in Section "Materials and Methods." (A) Induction of the las/rhl-dependent biosensor, determined by bioluminescence generation. The assay was performed using the $E$. coli JM109 reporter strain harboring the pSB1075 plasmid, which produces luminescence in response to 3-oxo- $\mathrm{C}_{12}-\mathrm{HSL}$. (B) Pyocyanin, (C) biofilm formation, (D) rhamnolipids, (E) polyhydroxyalkanoates, and (F) lipopolysaccharides. (D-F) Cell-free supernatants of 48-h grown cultures. Bars represent the mean \pm SE of three independent experiments. One-way ANOVA was performed, with Bonferroni post hoc test; $n=3$. Values for SE $\left(P<0.05^{\star},<0.001^{\star \star},<0.0001^{\star \star \star}\right)$ are shown.

bioluminescence in the QS reporter strain, using as a negative control the QS system double mutant PAO1-JM2 (lasI $\left.{ }^{-} / \mathrm{rhlI}^{-}\right)$ previously described (Li et al., 2007).

The cell-free supernatants of cultures obtained at $24 \mathrm{~h}$ from all the single and double mutants were able to induce a QSdependent bioluminescence of the reporter strain, with similar intensity as the WT PAO1 strain supernatant (Figure 5A). However, when supernatants of cultures grown for $48 \mathrm{~h}$ were tested, the fadD1Tn and fadD2Tn mutants showed a diminished luminescence intensity compared to the WT PAO1 strain, but the luminescence level induced by supernatants from the fadD4::Gm mutant did not change. Instead, the double mutants fadD1Tn/fadD2::Gm, fadD1Tn/fadD4::Gm, and $f a d D 2 T n / f a d D 4:: G m$ showed a diminished luminescence intensity compared to the WT strain. These findings indicate that the mutations in fadD1 and fadD2, but not in fadD4, affected 
the AHL-dependent las/rhl QS response, suggesting that the consequent modifications in the production of AHL-dependent virulence factors occurred on fadD1 and fadD2 mutants but did not occur in the fadD4::Gm mutant.

Virulence factors such as pyocyanin (Lau et al., 2004) and biofilm (Vital-Lopez et al., 2015; Thi et al., 2020) are important in bacterial-host colonization, and their production are dependent of QS regulation systems. Biofilm is a life mode that consists of microbes contained in slow-growing microcolonies embedded in a protective biopolymer matrix, which confers highest levels of resistance to antibiotics and the immune system. Biofilm is a common cause of persistent infections, being a key pathogen of chronic infections in the lungs of cystic fibrosis and immunocompromised patients (Moser et al., 2021). In $P$. aeruginosa, a biofilm is a protective structure for bacterial growth, essential for survival in nosocomial environments, and it may also maintain a persistence of the inflammatory response in chronic bacterial infections, favored by the excretion of diverse virulence factors; however, the degree of pathogenicity, expression of virulence factors, or resistance to antibiotic by bacteria cannot always be associated with the ability to form biofilms (Gajdács et al., 2021).

Findings showed that pyocyanin production in mutants was similar to that in the supernatants after 24-h growth; however, in supernatants at $48 \mathrm{~h}$, a significant increment of $\sim 60 \%$ was observed in the fadD1Tn single mutant, as well as in the double mutants fadD1Tn/fadD2::Gm and fadD1Tn/fadD4::Gm (Figure 5B). The amount of biofilm formed was significantly increased in the mutants fadD1Tn, fadD2Tn, fadD1Tn/fadD2::Gm, and fadD1Tn/fadD4::Gm with supernatants after 24-h growth. However, it remained similar between the WT and the fadD4::Gm mutant, while in the double mutant fadD2Tn/fadD4::Gm, the level of biofilm was diminished (Figure 5C). Interestingly, in supernatants from bacterial cultures grown for $48 \mathrm{~h}$, none of the mutants showed significant differences in biofilm production compared to the WT strain, except for the double mutant fadD2Tn/fadD4:: Gm, in which biofilm production was severely affected (Figure 5C). These results indicate that the $f a d D 1 / 2 / 4$-encoded proteins in $P$. aeruginosa PAO1 play important roles in biofilm production, probably associated with FA catabolism and energy availability.

Other QS-regulated virulence factors that are essential for host colonization of $P$. aeruginosa, which contain lipidic components in their structures, are RHL, PHA, and LPS (Pier, 2007; Reis et al., 2011; García-Reyes et al., 2020). In our study, we found that the RHL content was increased in the cell-free supernatants obtained at $48 \mathrm{~h}$ from cultures of the double mutants but was significantly impaired in the fadD2Tn mutant (Figure 5D).

In addition, it has been described that compounds that contain lipidic components such as PHA can be redirected from carbon storage to RHL synthesis (Gutiérrez-Gómez et al., 2019) and, in consequence, may increase the virulence and colonization capacity of the bacterium. Our results showed that the PHA content was unmodified in all $P$. aeruginosa strains, except in the double mutant fadD1Tn/fadD4::Gm, in which it was decreased by approximately $20 \%$ (Figure $5 \mathbf{E}$ ).
Interestingly, the LPS content was significantly increased in the fadD1Tn and fadD4::Gm mutants, as in the double mutants fadD1Tn/fadD4::Gm and fadD2Tn/fadD4::Gm (Figure 5F). These results indicate that the $P$. aeruginosa FadD4 is involved in the production, accumulation, and degradation of lipidcontaining cellular compounds such as biofilm, RHL, PHA, or LPS; consequently, these alterations may render different degrees of bacterial virulence.

\section{The fadD4 Mutation Increases Mortality of C. elegans Worms}

A diverse range of secreted molecules have been associated with the degree of bacterial virulence (Lee and Zhang, 2015; Valentini et al., 2018; Panayidou et al., 2020). A modification of the virulence factors observed in the fadD mutants reveals changes in the degree of pathogenicity of the PAO1 strain (Kang et al., 2010; Zarzycki-Siek et al., 2013). Thus, we investigated the effect of $f a d D$ mutations on the virulence of $P$. aeruginosa PAO1 cultures using the nematode in vivo virulence model.

Virulence was determined in C. elegans worms using cellfree supernatants of $P$. aeruginosa strains grown in LB medium (slow-killing assay) and live LB bacterial cultures (fast-killing assay). Worms exposed for $48 \mathrm{~h}$ to the supernatant from the fadD4::Gm single mutant collected from 24-h cultures exhibited survival kinetics similar to that of the fadD1Tn mutant and the PAO1 WT strain (Figure 6A), with a nematode survival of $\sim 30 \%$, compared with $\sim 45 \%$ for the WT strain. In contrast, worm survival increased to $\sim 60$ $70 \%$ in the case of double mutants fadD1Tn/fadD2::Gm, fadD1Tn/fadD4::Gm, and fadD2Tn/fadD4::Gm and the single mutant fadD2Tn (Figure 6A).

Because differential amounts of virulence factors were observed when using 24-h culture supernatants, the supernatants obtained at $48 \mathrm{~h}$ were also used to evaluate their effect on worm survival. The worm survival kinetics showed that supernatants from the single mutant fadD4::Gm and the double mutants fadD1Tn/fadD2::Gm and fadD2Tn/fadD4::Gm were more toxic than those from the WT strain and other mutants (Figure 6B). Additionally, the ability to kill C. elegans worms was tested with a fast-killing procedure. As shown in Figure 6C, among the $P$. aeruginosa strains tested for slow killing, the mutants fadD1Tn (24\% survival), fadD2Tn (12\% survival), and fadD4::Gm (35\% survival) were the most lethal to adult $C$. elegans worms. Double mutants fadD1Tn/fadD2::Gm (50\% survival), fadD1Tn/fadD4::Gm (52\% survival), and fadD2Tn/fadD4::Gm (51\% survival), as well as WT (58\% survival), were moderately less lethal in the first $36 \mathrm{~h}$ than others. The fadD4::Gm mutant showed a similar increase in lethality in both the slow- and fast-killing assays compared to the WT strain.

The induction of reactive oxygen species (ROS) has been described as the death mechanism associated with virulence factors (Lau et al., 2004); therefore, we used the worms C. elegans CL2166 to monitor ROS induction as an effect of the lethality of $P$. aeruginosa cultures. Images show a significant 

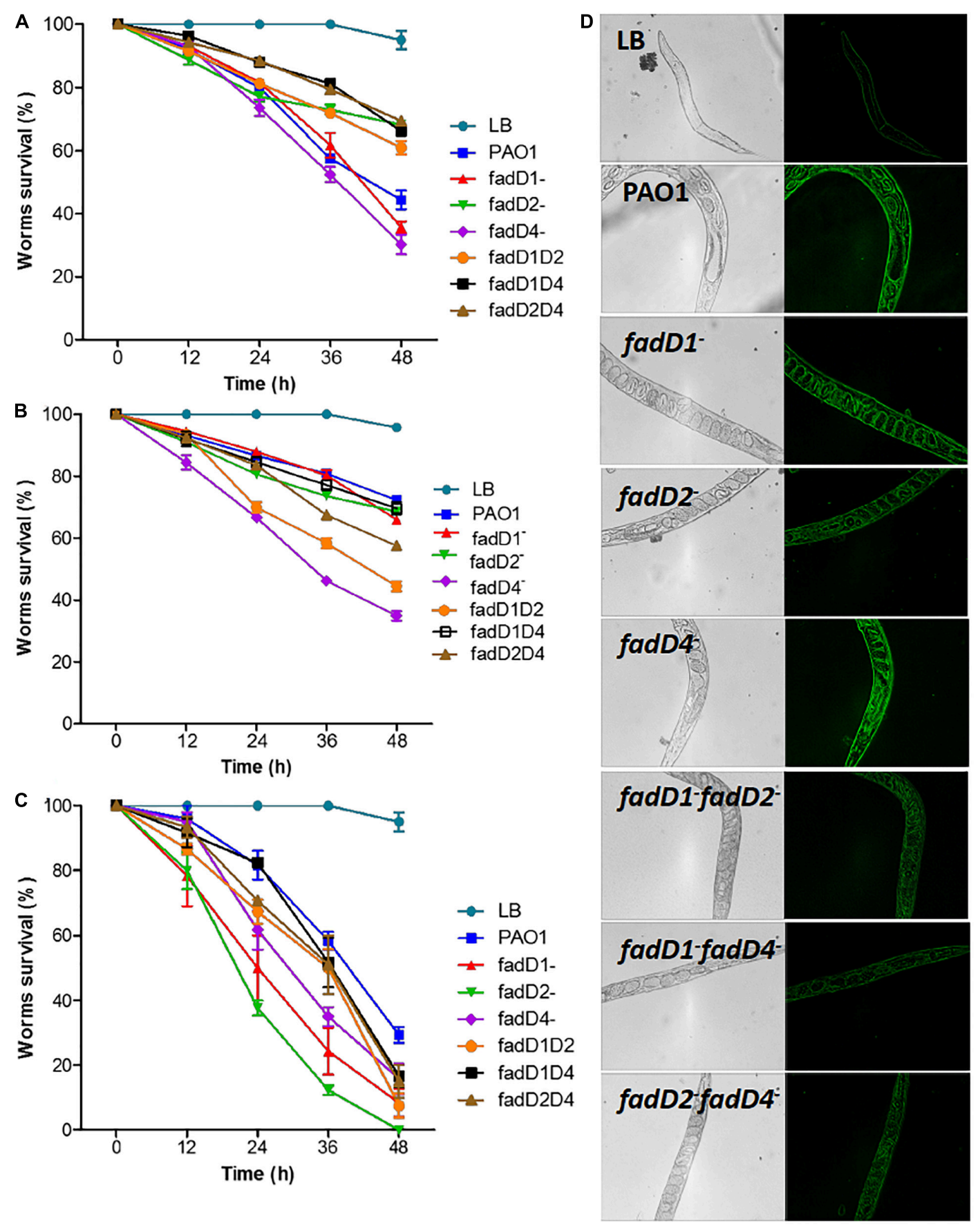

FIGURE 6 | Toxicity of $P$. aeruginosa PAO1 cultures on C. elegans worms. (A,B) Adult $C$. elegans worms (20 individuals) were incubated with cell-free supernatants obtained after growth of $P$. aeruginosa strains for $24 \mathrm{~h} \mathrm{(A)} \mathrm{and} 48 \mathrm{~h}$ (B) or with complete culture media (C). Worm survival was determined at indicated times as described in Section "Materials and Methods." Bars represent mean \pm standard error (SE) of three independent assays. (D) Images of oxidative stress responses of CL2166 worms to bacterial strains.

increase in the fluorescence of the worms exposed to 48h supernatants of cultures of the PAO1 WT and the single mutants fadD1Tn, fadD2Tn, and fadD4::Gm, while the ROS induction was minor in worms exposed to double mutants (Figure 6D). These results indicate that in the $C$. elegans model, ROS generation is not the main mechanism of virulence observed by the single $f a d D$ mutants of $P$. aeruginosa; however, ROS induction diminished in the double mutants, suggesting a possible implication in cytotoxicity. Thus, our findings confirm that the fadD1, fadD2, and fadD4 genes are implicated in the virulence of $P$. aeruginosa, suggesting that this genetic redundancy is a property that confers 


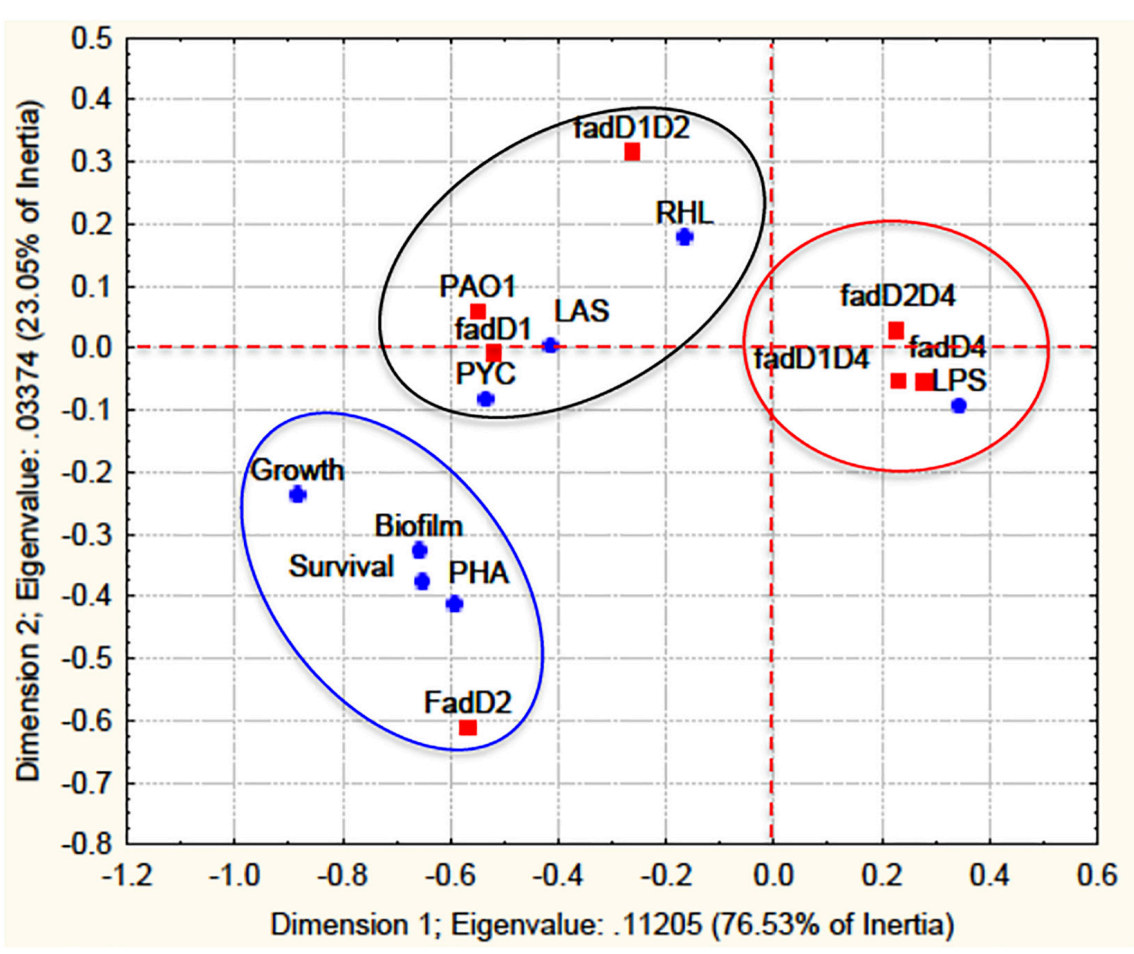

FIGURE 7 | A statistical correlation analysis of virulence factors with fadD mutants. A correlation analysis of virulence factor data from the mutagenesis approach was performed by multivariate exploratory techniques, with 2-D PCA correspondence analysis of frequencies with/without grouping variables using the STATISTICA Software System 8.0, Stat Soft. Inc. The fadD mutants are designated response variables (independent) and virulence factors as dependent variables. Survival, worm survival; Growth, growth in AT and FA; LAS, QS-las/rhl induction; PYC, pyocyanin; RHL, rhamnolipids; PHA, polyhydroxyalkanoates; LPS,

lipopolysaccharides; Biofilm, biofilm formation.

to this bacterium the ability to respond effectively during infection/pathogenesis processes on hosts or in colonization of ecological niches.

\section{Correlation Between fadD Mutants and Virulence Factors}

Quantitative values of all virulence factors determined in the fadD mutants were analyzed using a statistical correlation analysis between genotypes and phenotypes. Based on the correlation analysis, the virulence factors were classified into three groups according to the dependent variables (Figure 7). The fadD1Tn and $f a d D 1 T n / f a d D 2:: G m$ mutants were grouped with the PAO1 WT strain and correlated with the virulence factors AHLdependent luminescence, rhamnolipids, and pyocyanin (black circle in Figure 7). The second group correlated the single mutant fadD2Tn with growth on fatty acids, biofilm production, polyhydroxyalkanoate content, and worm survival (blue circle in Figure 7). Interestingly, the third group correlated the single mutant fadD4::Gm and its respective double mutants $f a d D 1 T n / f a d D 4:: G m$ and $f a d D 2 T n / f a d D 4:: G m$ with the response factor, LPS content (red circle in Figure 7). This analysis indicates the potential dependence between LPS content and the fadD4 mutation, suggesting the possible participation of LPS in the increased degree of pathogenicity in the fadD4 mutant in the worm survival assay.

\section{The fadD4 Mutation Modifies Lipopolysaccharides Content and
Structure} Lipopolysaccharides are components of the outer membrane of Gram-negative bacteria and are considered to be important virulence factors related to acute $P$. aeruginosa infections and pathogenesis (Lam et al., 2011; Cullen et al., 2015). Modulation of LPS synthesis and structure results in immune evasion, persistent inflammation, and antimicrobial resistance and is important in the adaptation of opportunistic pathogens such as $P$. aeruginosa to chronic infections in the respiratory system (Cullen et al., 2015; Moser et al., 2021). In addition, the LPS structure can be modified at the level of lipid A or antigen $\mathrm{O}$ as described for bacteriophage-resistant $P$. aeruginosa PAO1 (Latino et al., 2017).

Thus, we determined the LPS content and characterized the nature of its lipidic component to elucidate FadD4 participation in their synthesis/degradation and correlation with P. aeruginosa pathogenicity.

As described above, the LPS content (determined by measuring disaccharide content) was increased in the fadD1Tn and fadD4::Gm mutants, as well as in the double mutants fadD1Tn/fadD4::Gm and fadD2Tn/fadD4::Gm (Figure 5F). An analysis of LPS isolated from bacterial pellets on polyacrylamide gels and silver staining (Latino et al., 2017) revealed a significant increase in the amount of LPS in the 
double mutant fadD1Tn/fadD4::Gm and a minor increase in the fadD1Tn/fadD2::Gm and fadD2Tn/fadD4::Gm mutants (Figure 8A). In agreement, when the LPS samples were isolated by a different method (Mirzaei et al., 2011) and analyzed on gels under conditions that focused on the LPS-O antigen, gel images showed that the mutant fadD4::Gm and the double mutants fadD1Tn/fadD4::Gm and fadD2Tn/fadD4::Gm contained increased amounts of LPS compared to the WT and other mutants, as well as differential patterns of bands between them (Figure 8B). These findings indicate that in addition to the increased LPS content in the fadD4::Gm and fadD1Tn/fadD4::Gm mutant, the LPS structure could be modified at the level of lipid A (lipidic component of LPS) or antigen O (carbohydrate component of LPS).

Modifications in the acyl groups of lipid A have been described to occur in LPS of $P$. aeruginosa strains from diverse sources (Lam et al., 2011). To elucidate whether LPS is modified at the level of lipid A structure, the LPS isolated from the $P$. aeruginosa strains were analyzed based on the fatty acid content of lipid A by quantification of derived FAMEs from LPS. The FAME content was significantly increased in the fadD $4:: G m$ mutant, as well as its double mutants fadD1Tn/fadD4::Gm and fadD2Tn/fadD4::Gm, in comparison to the WT strain (Figure 8C), confirming an increase in the LPS content in agreement with LPS quantitation by saccharide determination and gel observation (Figures $\mathbf{5 F}$, $\mathbf{8 A}, \mathbf{B})$. Considering the nature of the fatty acids contained in the lipid A of LPS, the analyzed proportion of FAMEs showed that the FAs with chain lengths $\mathrm{C}_{16}, \mathrm{C}_{12}, \mathrm{C}_{10}$, and $\mathrm{C}_{8}$ were modified in the LPS from the fadD4::Gm mutant, as well as in the double mutants, compared with the LPS from PAO1 WT (Figures 8C,D). These data confirm that the structure of LPS was modified at the level of the acyl group content in the structure of the lipid A component. Additionally, the quantitation of PHA by FAME determination showed that only the fadD1 mutant contained decreased amounts of alkanoic acids, and a slight modification of alkanoic acid proportions was observed in the PHA from mutants (Figures 8E,F). Therefore, these results suggest that in $P$. aeruginosa PAO1, the LPS content and, importantly, the acyl groups of FA content in lipid A were modified by the effect of the fadD 4 mutation, which could be associated with the increase in toxicity of the cultures and supernatants observed in the worm survival assays.

\section{The fadD4 Mutation Modifies the Pyrogenic Response to Lipopolysaccharides in Rats}

Lipopolysaccharides (intraperitoneally administered) is known to be a pyrogenic agent in animals, consisting of $O$-polysaccharide, R-core, and lipid A components, each of which can stimulate the innate immune response. In this sense, structural modifications on lipid A are observed in the number, position, the nature of the linked acyl groups, and on phosphate groups (Pier, 2007; Lam et al., 2011; Moser et al., 2021). Pyrogenic properties associated with the lipid A moiety of LPS are transduced by the TLR4/MD2 receptor of the mammalian innate immune system (Behzadi et al., 2021b). On the other hand, picomolar levels of lipid A induce macrophages to synthesize potent mediators of inflammation, such as TNF- $\alpha$ and IL- $1 \beta$ (Zasłona et al., 2017). In $P$. aeruginosa, the conserved lipid A unit is a bisphosphorylated disaccharide of glucosamine, typically with four to seven acyl chains, and has been also described to consist of four to six FA acyl chains (Simpson and Trent, 2019). Modifications of lipid A from LPS affect several physiological processes in Gram-negative bacteria (e.g., effects on the permeability of the outer membrane, recognition by immune cells, and antimicrobial resistance). Many bacteria utilize lipid A modifications to evade recognition by the mammalian immune response (Simpson and Trent, 2019), and studies have indicated that the number and carbon chain length of acyl groups of FA are critical for TLR4 activation, which can modify the magnitude of the immune response (Harada et al., 1994; McIsaac et al., 2012; Lo Sciuto et al., 2019). Complementary to FA degradation, mutations in enzymes involved in de novo FA biosynthesis, such as FabF1/F2 from Rhizobium leguminosarum, modify the content and type of FA from the lipid A of LPS and consequently modify adaptability and colonization (Vanderlinde et al., 2009).

To confirm that the mutation in the fadD4 gene modifies the lipid A structure and, consequently, correlates with the degree of bacterial pathogenicity associated with LPS content and structural composition, a systemic inflammation model in rodents to test the LPS immunogenicity was carried out. The body temperature of the animals administered with LPS at the same concentration increased with time, as can be observed in the WT strain, in which the linear correlation for the PAO1 WT-LPS showed a value of slope $=0.00951$ (Figure 9A). A similar increase was observed in the fadD4::Gm mutant (slope $=0.00952$ ), while a moderate increase in temperature (lower slope) was observed for the single mutants fadD1Tn (slope $=0.00786$ ) and fadD2Tn (slope $=0.00755$ ) and the double mutant fadD1Tn/fadD2::Gm (slope $=0.00791$ ). Interestingly, animals injected with LPS from the double mutants fadD1Tn/fadD4::Gm and $f a d D 2 T n / f a d D 4:: G m$ showed a minor increase in body temperature, with slopes of 0.00704 and 0.00713 , respectively (Figure 9A). In addition, all the rats treated with LPS presented similar symptoms: less active, disinclined to feed, piloerection, hunched posture, ataxic, and weak, thus demonstrating that LPS induced systemic inflammation in animals, with the effect of the fadD mutations being associated with this response.

\section{The fadD4 Mutation Modifies the Antigenic Response to Lipopolysaccharides in Rats}

In the mammalian inflammatory response, interleukin-8 (IL-8) is a chemotactic leukocyte-activating cytokine produced by various types of cells upon stimulation with inflammatory stimuli and exerting a variety of functions on leukocytes of the neutrophil type (Harada et al., 1994).

In our rodent inflammation model, an analysis of leucocyte proportions in the blood of the LPS-treated rats showed that LPS from the fadD4::Gm mutant slightly decreased the number of neutrophils, but increased the proportion of monocytes and eosinophils in the bloodstream, compared to the WT strain 


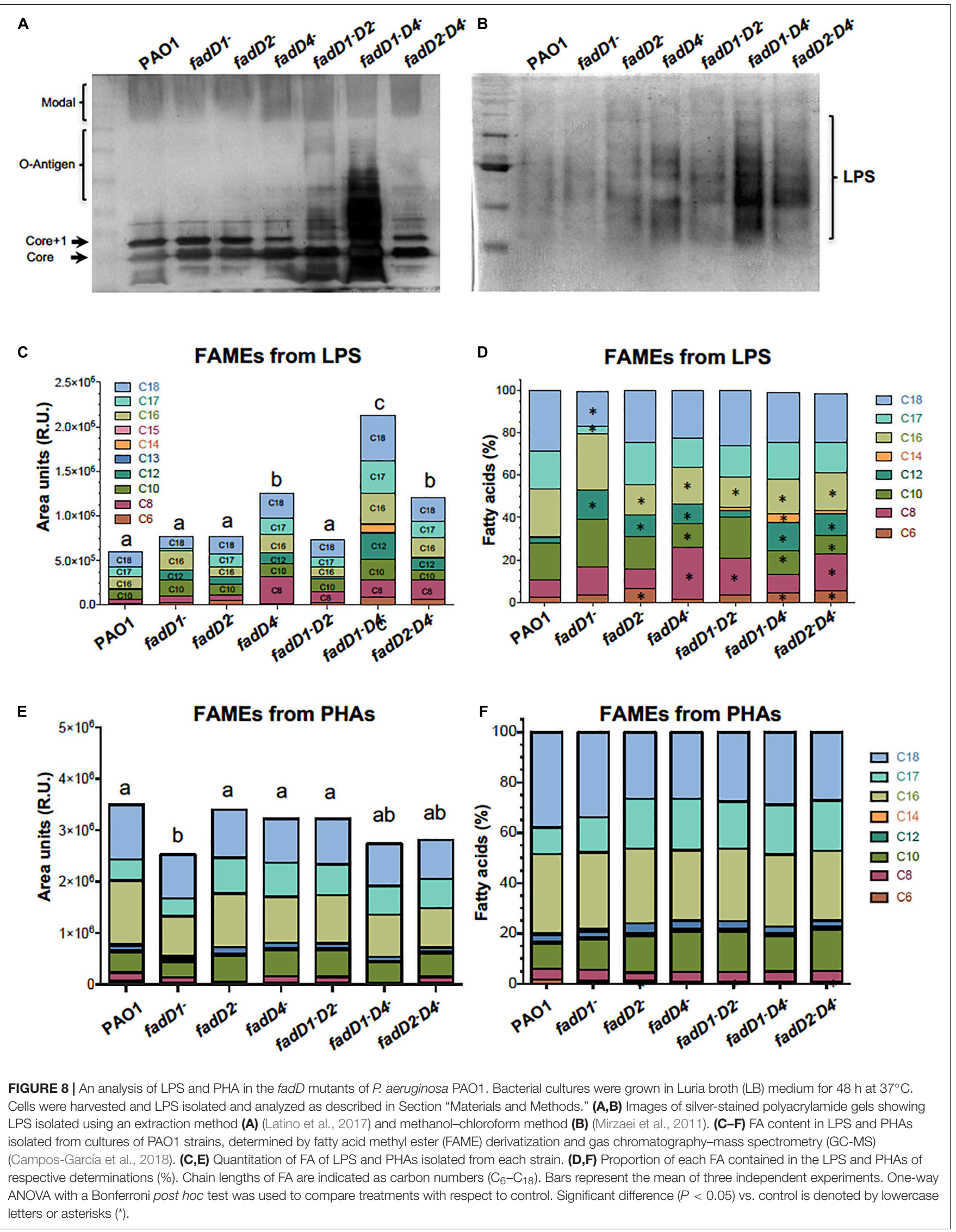


A

\section{Body temperature in rats injected with LPS from $P$. aeruginosa strains}

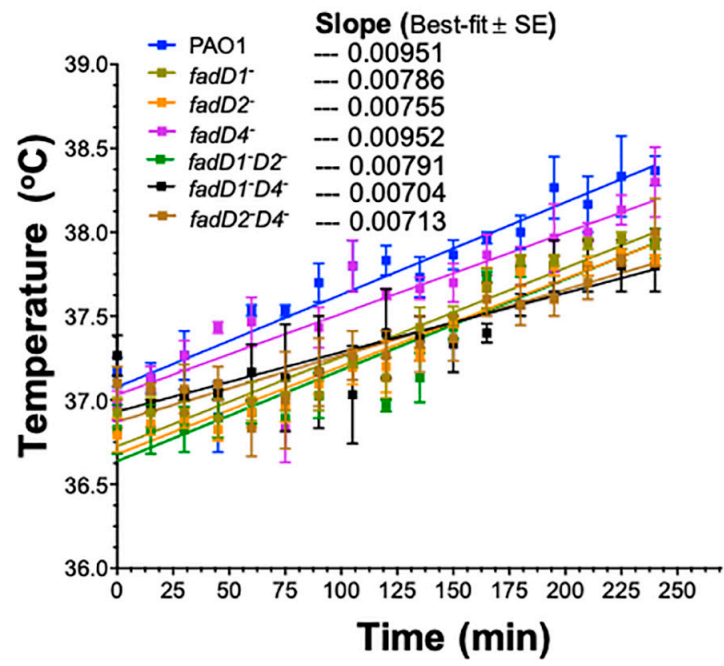

B

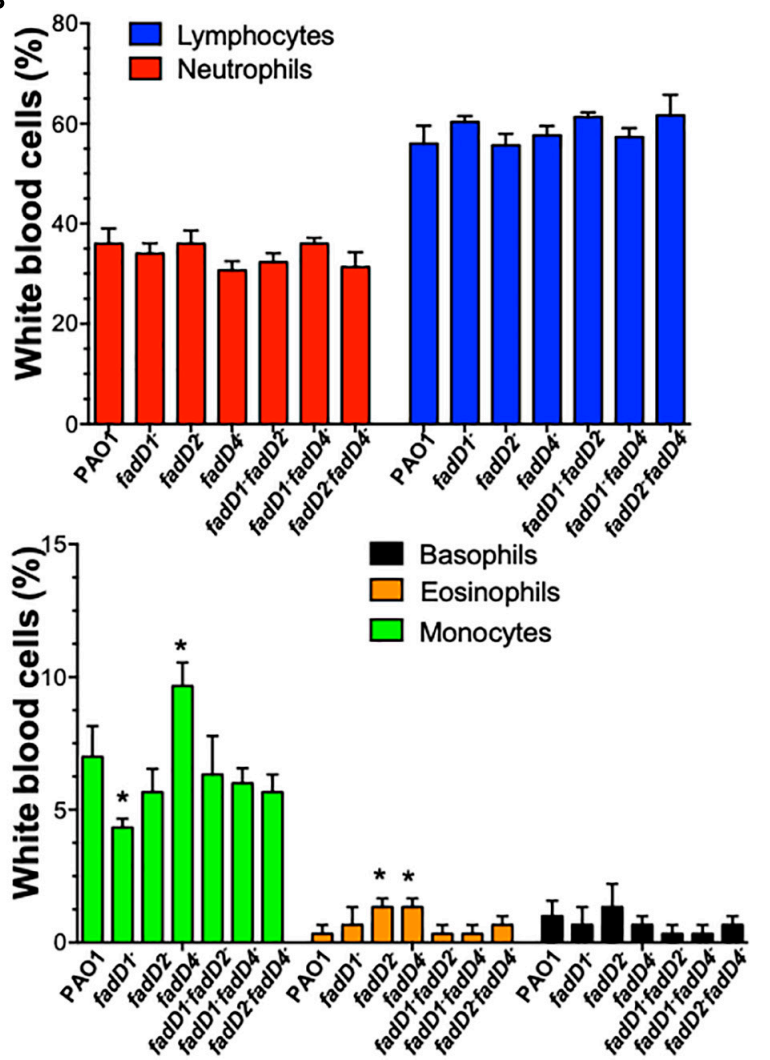

C
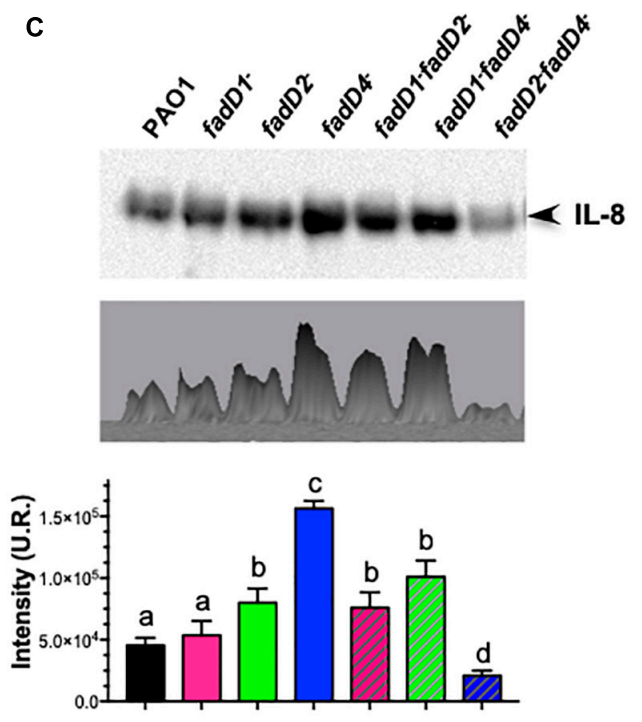

D
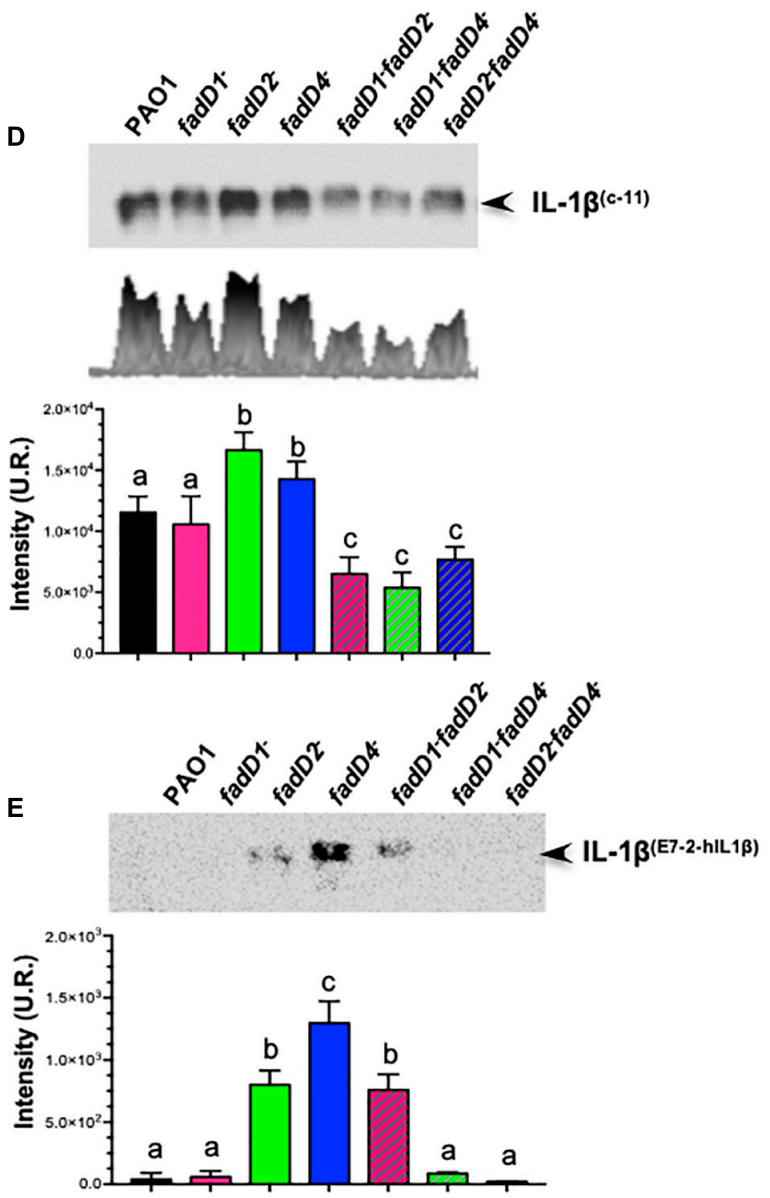

FIGURE 9 | The effect of LPS from fadD mutants of $P$. aeruginosa PAO1 on body temperature in rats and immunodetection of interleukins. (A) Change in temperature in Wistar rats in response to intraperitoneal injections of LPS isolated from the $P$. aeruginosa PAO1 strains. A dose of $50 \mu \mathrm{g} / \mathrm{kg}$ induced fever in all rats after injection $(n=3)$. Lineal correlation curves are shown, and values of slopes of best fit are indicated. (B) Determination of proportions of white blood cells in rats treated with LPS samples. (C-E) Immunodetection of interleukins in the serum of rats after LPS treatment: (C) IL-8 using anti-IL-8 antibody (sc376750, Santa Cruz

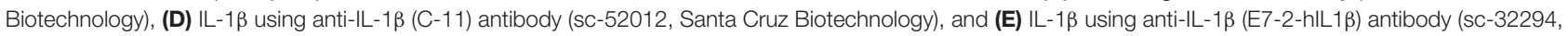
Santa Cruz Biotechnology). Immunodetection of interleukins on western blot membranes was analyzed by densitometry using the Image Lab 6.0 .1 software. One-way ANOVA with a Bonferroni post hoc test was used to compare treatments with respect to control. Significant difference $(P<0.05)$ vs. control is denoted by lowercase letters or asterisks $\left(^{*}\right)$. 


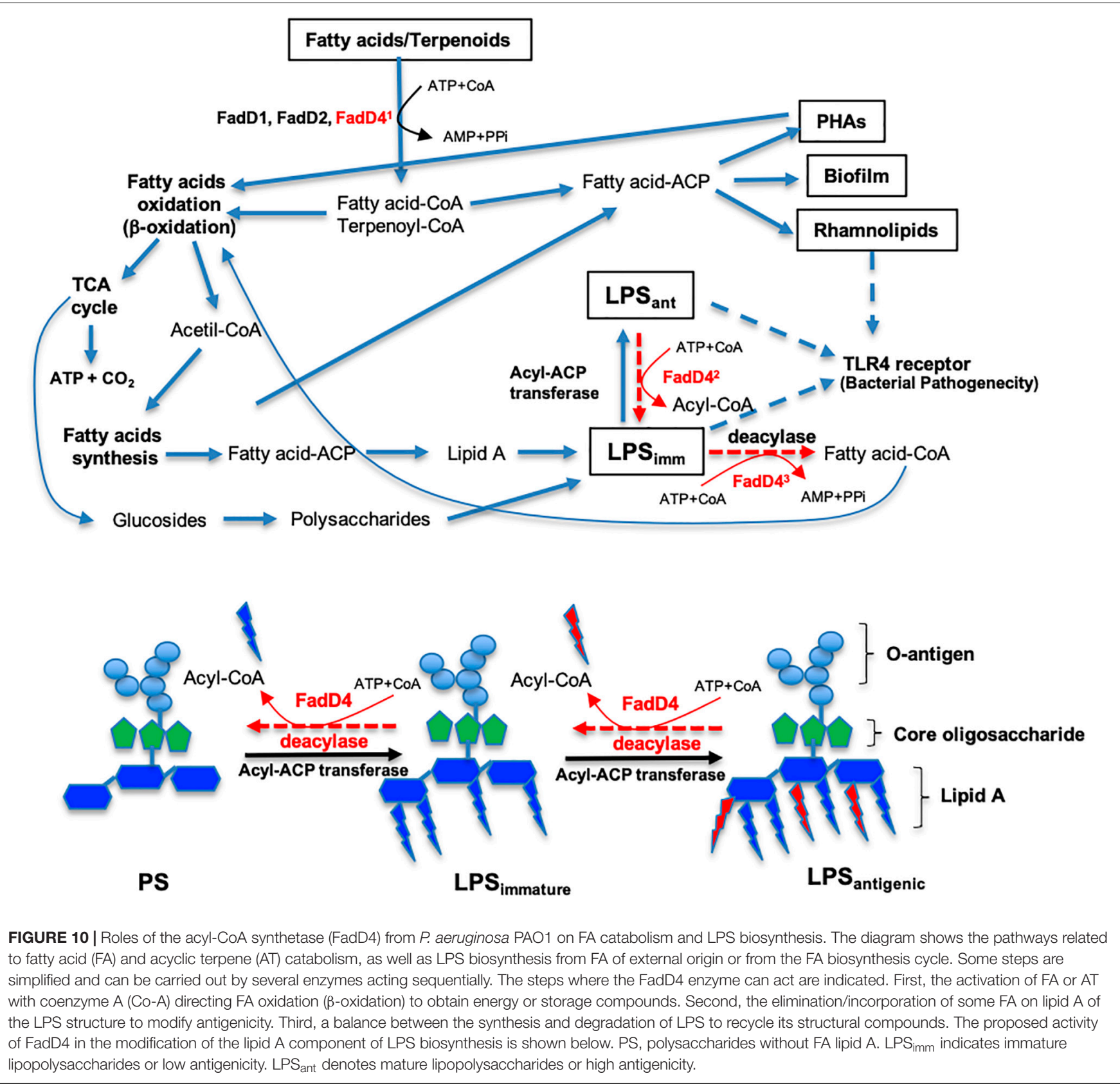

(Figure 9B). In addition, a western blotting analysis of the blood serum of the LPS-treated rats showed that the LPS from all strains were capable of inducing secretion/production of IL-8 at differential levels, with the single mutants fadD2 and fadD4 presenting significantly increased production of IL8 compared to the LPS from the WT strain (Figure 9C). LPS from the fadD4 mutant induced the highest level of IL-8. The double mutants fadD1Tn/fadD2::Gm and fadD1Tn/fadD4::Gm also increased the expression levels of IL-8; however, the double mutant fadD2Tn/fadD4::Gm induced lower levels of IL-8 secretion/production than all other strains.

It well known that bacterial LPS also induces the secretion/production of IL- $1 \beta$ in addition to IL- 8 , which is produced in response to LPS as well as TNF- $\alpha$ (Zasłona et al., 2017). An analysis of IL- $1 \beta$ production in the serum of LPStreated animals (Figures 9D,E) revealed higher IL-1 $\beta$ levels in the case of fadD2 and fadD4 mutants, but interestingly, IL-1 $\beta$ levels were diminished when LPS from double mutants were used (Figure 9D). Interestingly, when another anti-IL-1 $\beta$ antibody was used, the IL-1 $\beta$ immunodetection was mainly observed in the serum of LPS-treated animals from the fadD4 mutant (Figure 9E). These findings indicate that the LPS from the fadD mutants differentially induced the immune response (determined by IL- 8 and IL- $1 \beta$ secretion/production), dependent on the TLR4/MD2 response transductor. Our findings indicate that the FadD2 and FadD4 acyl-CoA synthetases modify the 
induction of innate immune response associated with LPS content and lipid A structural conformation. The different acyl groups of the FA composition of lipid A on these mutants indicate that the antigenicity can be differentially modulated, as observed in the IL- 8 versus IL-1 $\beta$ induction (Figures $9 \mathrm{C}-\mathbf{E}$ ), suggesting that the acyl-CoA synthetases FadD2 and FadD4 are mainly involved in LPS synthesis and determine the structural conformation of lipid A, whose antigenicity through the induction of inflammatory mediators IL- 8 and IL- $1 \beta$ has been associated with transduction of response by the TLR4/MD2 receptor of the mammalian immune system.

The pathogenesis of $P$. aeruginosa associated to TLR4 sharing ligands with TLR2 recognizes LPS by binding to the lipid A component on the outer membrane of bacteria. In cystic fibrosis patients, $P$. aeruginosa produces several different varieties of lipid A, contributing with the degree of virulence, which is associated to hexa-acylated lipid $\mathrm{A}$, the more potent agonist to TLR4 receptor (McIsaac et al., 2012; Behzadi et al., 2021b). About the mechanism bases of signal transduction, TLR4-MD2 heterodimers are the functional receptors of bacterial LPS. Acyl chains within the lipid A region of LPS interact with distinct regions of two TLR4-MD2 heterodimers, where five of the six acyl chains present in lipid A interact with a hydrophobic pocket present in the MD2 component of a TLR4-MD2 heterodimer. In other cases, LPS structures that contain less than six acyl chains in the lipid A have weakened inflammatory activities by interfering with the TLR4-MD2 heterodimer structuration (McIsaac et al., 2012).

In our study, we present elements that contribute to elucidating the role that can be associated with the fadD gene redundancy in $P$. aeruginosa $\mathrm{PAO} 1$ and their participation in determining the structure and composition of lipid A. As is well known, FA as substrates differentially induce the expression of fadD genes and consequently may modify the LPS antigenicity associated with their content and structure. Findings showed that fadD2 and fadD4 modified the LPS content and FA content of lipid A, producing differential modulation of LPS antigenicity. In this context, the LPS from the fadD1 and fadD2 mutants were less antigenic and pyrogenic in rats than from the WT strain; however, the LPS from the fadD4 mutant was more antigenic than that from the WT. In agreement, LPS from the fadD2 and fadD4 mutants were better inducers of proinflammatory cytokines, IL-1 $\beta$ and IL-8, than the WT. The differential effects by LPS isolated from the fadD mutants could be explained in agreement to the roles that the IL-1 (IL- $1 \alpha$ and IL- $1 \beta$ forms) plays in the regulation of immune response and inflammation, acting as an activator of $\mathrm{T}$ and $\mathrm{B}$ lymphocytes and natural killer cells, which require IL-1 $\beta$ for production of the antipathogen IFN- $\gamma$. In addition, differential induction of immune response may suggest the participation of different pathways such as the activation of the Nod-like receptor 3 (NLRP3) inflammasome, which is important for the activation of innate immune response to some DAMPs and PAMPs (Behzadi et al., 2021b; Lin et al., 2021).

Finally, we propose that the pathogenicity of $P$. aeruginosa PAO1 by FadD4 acyl-CoA synthetase involves the bioavailability of substrates such as acyclic terpene and fatty acids as carbon and energy sources by a mechanism of ATP-dependent acylCoA activation (Figure 10). This process allows $P$. aeruginosa to produce energy and metabolites via FA oxidation ( $\beta$-oxidation pathway) and tricarboxylic acid cycle. These components can be subsequently utilized for de novo FA synthesis to produce storage energy compounds such as polyhydroxyalkanoates and structural components such as rhamnolipids, biofilm, and LPS, where some of the components are composed of acyl groups of FA. Our study demonstrates that FadDs participate in these processes, but more importantly, FadD4 acyl-CoA synthetase, which was involved in the activation of acyl-FA, was directed to LPS biosynthesis (Figure 10). In addition, FadD4 may be suggested to contribute with modification of the FA composition of the lipid A component during LPS biosynthesis, which renders a differential degree of virulence and antigenicity of the P. aeruginosa PAO1 LPS. This structural modification associated to FadD4 can also be involved in the elimination or substitution of some acylFA on lipid A in the LPS structure, which in turn modifies their antigenicity (Figure 10; see LPS imm $_{\text {and }}$ LPS $_{\text {ant }}$ types, indicated as LPS with low and strong antigenicity, respectively). Another possibility suggests that FadD4 activity is involved in maintaining the balance between LPS synthesis and degradation, which is important for recycling the lipidic components of LPS. Thus, our study contributes to a deeper understanding of the mechanism involved in the pathogenicity of Gram-negative bacteria, highlighting the importance of lipid A modifications on the LPS virulence factor and the induced immune response in the host by a TLR4-dependent manner.

\section{CONCLUSION}

Our work confirms that fadD4 encodes a terpenoyl-CoA synthetase, which is essential for acyclic terpene assimilation and capable of activating fatty acids, similar to the FadD1 and FadD2 homologous enzymes. In addition, FadD4 plays important roles in the synthesis of LPS at the level of the lipid A component, by modifying the acyl groups of FA contained in the lipid A and consequently the amounts of LPS. The structural modification of lipid A was reflected in the degree of virulence of the $P$. aeruginosa PAO1 associated mainly with LPS in a rat model of induction of immune response, which was mediated by the IL- 8 and IL- $1 \beta$ interleukins transduced by the TLR4/MD2 receptor.

\section{DATA AVAILABILITY STATEMENT}

The original contributions presented in the study are included in the article/Supplementary Material, further inquiries can be directed to the corresponding author.

\section{ETHICS STATEMENT}

The animal study was reviewed and approved by Institutional Committee for Use of Animals of the I.I.Q.B/Universidad Michoacana de San Nicolás de Hidalgo in agreement with NOM062-ZOO-1999, Ministry of Agriculture of Mexico. 


\section{AUTHOR CONTRIBUTIONS}

JC-G conceptualized and designed the study. LM-A, GO, and AD-P developed the methodology. LM-A and JC-G analyzed and interpreted the data. LM-A, JV, HR-D, EG-P, and JC-G wrote, reviewed, and/or revised the manuscript. JV, HR-D, EG-P, and JC-G provided administrative, technical, or material support. JC-G supervised the study. All authors contributed to the article and approved the submitted version.

\section{FUNDING}

This study received funding from the Consejo Nacional de Ciencia y Tecnologia (CONACYT-México, Grant No. 256119), Marcos Moshinsky Foundation (grant 2014-16), and Universidad

\section{REFERENCES}

Aguilar, J. A., Zavala, A. N., Diaz-Perez, C., Cervantes, C., Diaz-Perez, A. L., and Campos-Garcia, J. (2006). The atu and liu clusters are involved in the catabolic pathways for acyclic monoterpenes and leucine in Pseudomonas aeruginosa. Appl. Environ. Microbiol. 72, 2070-2079. doi: 10.1128/aem.72.3.2070-2079. 2006

Behzadi, P., Baráth, Z., and Gajdács, M. (2021a). It's not easy being green: a narrative review on the microbiology, virulence and therapeutic prospects of multidrug-resistant Pseudomonas aeruginosa. Antibiotics (Basel) 10:10010042. doi: 10.3390/antibiotics10010042

Behzadi, P., García-Perdomo, H. A., and Karpiński, T. M. (2021b). Tolllike receptors: general molecular and structural biology. J. Immunol. Res. 2021:9914854. doi: 10.1155/2021/9914854

Brimacombe, C. A., Stevens, A., Jun, D., Mercer, R., Lang, A. S., and Beatty, J. T. (2013). Quorum-sensing regulation of a capsular polysaccharide receptor for the Rhodobacter capsulatus gene transfer agent (RcGTA). Mol. Microbiol. 87, 802-817. doi: $10.1111 / \mathrm{mmi} .12132$

Calisti, C., Ficca, A. G., Barghini, P., and Ruzzi, M. (2008). Regulation of ferulic catabolic genes in Pseudomonas fluorescens BF13: involvement of a MarR family regulator. Appl. Microbiol. Biotechnol. 80, 475-483. doi: 10.1007/s00253008-1557-4

Campos-García, J. (2010). "Metabolism of acyclic terpenes by Pseudomonas," in Pseudomonas: Volume 6: Molecular Microbiology, Infection and Biodiversity, eds L. J. Ramos and A. Filloux (Dordrecht: Springer), 235-253. doi: 10.1007/ 978-90-481-3909-5_8

Campos-García, J., Caro, A. D., Nájera, R., Miller-Maier, R. M., Al-Tahhan, R. A., and Soberón-Chávez, G. (1998). The Pseudomonas aeruginosa rhlG gene encodes an NADPH-dependent $\beta$-ketoacyl reductase which is specifically involved in rhamnolipid synthesis. J. Bacteriol. 180, 4442-4451. doi: 10.1128/ JB.180.17.4442-4451.1998

Campos-García, J., and Soberón-Chávez, G. (2000). Degradation of the methyl substituted alkene, citronellol, by Pseudomonas aeruginosa, wild type and mutant strains. Biotechnol. Lett. 22, 235-237.

Campos-García, J., Vargas, A., Farías-Rosales, L., Miranda, A. L., MezaCarmen, V., and Díaz-Pérez, A. L. (2018). Improving the organoleptic properties of a craft mezcal beverage by increasing fatty acid ethyl ester contents through ATF1 expression in an engineered Kluyveromyces marxianus UMPe-1 yeast. J. Agric. Food Chem. 66, 4469-4480. doi: 10.1021/acs.jafc. $8 \mathrm{~b} 00730$

Chavez-Aviles, M., Diaz-Perez, A. L., Reyes-de la Cruz, H., and Campos-Garcia, J. (2009). The Pseudomonas aeruginosa liuE gene encodes the 3-hydroxy3-methylglutaryl coenzyme a lyase, involved in leucine and acyclic terpene catabolism. FEMS Microbiol. Lett. 296, 117-123. doi: 10.1111/j.1574-6968.2009. 01624.x
Michoacana de San Nicolás de Hidalgo (grant CIC-2.14). The funders were not involved in the study design; collection, analysis, and interpretation of data; the writing of this article; or the decision to submit it for publication.

\section{ACKNOWLEDGMENTS}

We thank Pseudomonas Genome Center Annotation and UWGC for PAO1-transposon mutants donation.

\section{SUPPLEMENTARY MATERIAL}

The Supplementary Material for this article can be found online at: https://www.frontiersin.org/articles/10.3389/fmicb. 2021.785112/full\#supplementary-material

Coffey, B. M., and Anderson, G. G. (2014). "Biofilm formation in the 96-Well microtiter plate," in Pseudomonas Methods and Protocols, eds A. Filloux and J.-L. Ramos (New York, NY: Springer), 631-641.

Corpet, F. (1988). Multiple sequence alignment with hierarchical clustering. Nucleic Acids Res. 16, 10881-10890. doi: 10.1093/nar/16.22. 10881

Cullen, L., Weiser, R., Olszak, T., Maldonado, R. F., Moreira, A. S., Slachmuylders, L., et al. (2015). Phenotypic characterization of an international Pseudomonas aeruginosa reference panel: strains of cystic fibrosis (CF) origin show less in vivo virulence than non-CF strains. Microbiology (Reading) 161, 1961-1977. doi: 10.1099/mic.0.000155

Díaz-Pérez, A. L., Díaz-Pérez, C., and Campos-García, J. (2015). Bacterial l-leucine catabolism as a source of secondary metabolites. Rev. Environ. Sci. Bio Technol. $15,1-29$.

Diaz-Perez, A. L., Nunez, C., Meza Carmen, V., and Campos-Garcia, J. (2018). The expression of the genes involved in leucine catabolism of Pseudomonas aeruginosa is controlled by the transcriptional regulator LiuR and by the CbrAB/Crc system. Res. Microbiol. 169, 324-334. doi: 10.1016/j.resmic.2018. 05.004

Diaz-Perez, A. L., Zavala-Hernandez, A. N., Cervantes, C., and Campos-Garcia, J. (2004). The gnyRDBHAL cluster is involved in acyclic isoprenoid degradation in Pseudomonas aeruginosa. Appl. Environ. Microbiol. 70, 5102-5110. doi: 10.1128/aem.70.9.5102-5110.2004

Feng, S., Xu, C., Yang, K., Wang, H., Fan, H., and Liao, M. (2017). Either fadD1 or fadD2, which encode acyl-CoA synthetase, is essential for the survival of Haemophilus parasuis SC096. Front. Cell Infect. Microbiol. 7:72. doi: 10.3389/ fcimb.2017.00072

Figurski, D. H., and Helinski, D. R. (1979). Replication of an origin-containing derivative of plasmid RK2 dependent on a plasmid function provided in trans. Proc. Natl. Acad. Sci. U.S.A. 76, 1648-1652. doi: 10.1073/pnas.76.4. 1648

Forster-Fromme, K., Hoschle, B., Mack, C., Bott, M., Armbruster, W., and Jendrossek, D. (2006). Identification of genes and proteins necessary for catabolism of acyclic terpenes and leucine/isovalerate in Pseudomonas aeruginosa. Appl. Environ. Microbiol. 72, 4819-4828. doi: 10.1128/aem.00 853-06

Gajdács, M., Baráth, Z., Kárpáti, K., Szabó, D., Usai, D., Zanetti, S., et al. (2021). No correlation between biofilm formation, virulence factors, and antibiotic resistance in Pseudomonas aeruginosa: results from a laboratorybased in vitro study. Antibiotics (Basel) 10:1134. doi: 10.3390/antibiotics100 91134

García-Reyes, S., Soberón-Chávez, G., and Cocotl-Yanez, M. (2020). The third quorum-sensing system of Pseudomonas aeruginosa: Pseudomonas quinolone signal and the enigmatic PqsE protein. J. Med. Microbiol. 69, 25-34. doi: 10.1099/jmm.0.001116 
Gonzalez, O., Ortiz-Castro, R., Diaz-Perez, C., Diaz-Perez, A. L., Magana-Duenas, V., Lopez-Bucio, J., et al. (2017). Non-ribosomal peptide synthases from Pseudomonas aeruginosa play a role in cyclodipeptide biosynthesis, quorumsensing regulation, and root development in a plant host. Microb. Ecol. 73, 616-629. doi: 10.1007/s00248-016-0896-4

Gutiérrez-Gómez, U., Servín-González, L., and Soberón-Chávez, G. (2019). Role of $\beta$-oxidation and de novo fatty acid synthesis in the production of rhamnolipids and polyhydroxyalkanoates by Pseudomonas aeruginosa. Appl. Microbiol. Biotechnol. 103, 3753-3760. doi: 10.1007/s00253-01909734-x

Harada, A., Sekido, N., Akahoshi, T., Wada, T., Mukaida, N., and Matsushima, K. (1994). Essential involvement of interleukin-8 (IL-8) in acute inflammation. J. Leukoc Biol. 56, 559-564. doi: 10.1002/jlb.56.5.559

Holtzapple, E., and Schmidt-Dannert, C. (2007). Biosynthesis of isoprenoid wax ester in Marinobacter hydrocarbonoclasticus DSM 8798: identification and characterization of isoprenoid coenzyme A synthetase and wax ester synthases. J. Bacteriol. 189, 3804-3812. doi: 10.1128/JB.019 32-06

Hume, A. R., Nikodinovic-Runic, J., and O'Connor, K. E. (2009). FadD from Pseudomonas putida CA-3 is a true long-chain fatty acyl coenzyme a synthetase that activates phenylalkanoic and alkanoic acids. J. Bacteriol. 191, 7554-7565. doi: 10.1128/JB.01016-09

Jacobs, M. A., Alwood, A., Thaipisuttikul, I., Spencer, D., Haugen, E., Ernst, S., et al. (2003). Comprehensive transposon mutant library of Pseudomonas aeruginosa. Proc. Nat. Acad. Sci. U.S.A. 100, 14339-14344. doi: 10.1073/pnas.203628 2100

Kang, Y., Zarzycki-Siek, J., Walton, C. B., Norris, M. H., and Hoang, T. T. (2010). Multiple FadD acyl-CoA synthetases contribute to differential fatty acid degradation and virulence in Pseudomonas aeruginosa. PLoS One 5:e13557. doi: 10.1371/journal.pone.0013557

King, J. D., Mulrooney, E. F., Vinogradov, E., Kneidinger, B., Mead, K., and Lam, J. S. (2008). IfnA from Pseudomonas aeruginosa $\mathrm{O} 12$ and wbuX from Escherichia coli O145 encode membrane-associated proteins and are required for expression of 2,6-dideoxy-2-acetamidino-L-galactose in lipopolysaccharide O antigen. J. Bacteriol. 190, 1671-1679. doi: 10.1128/JB.01 708-07

Kokotek, W., and Lotz, W. (1991). Construction of a mobilizable cloning vector for site-directed mutagenesis of gram-negative bacteria: application to Rhizobium leguminosarum. Gene 98, 7-13. doi: 10.1016/0378-1119(91) 90097-u

Kozak, W., Kluger, M., Soszynski, D., Conn, C., Rudolph, K., Leon, L., et al. (2006). IL-6 and IL-1beta in fever: studies using cytokine-deficient (knockout) micea. Ann. N.Y. Acad. Sci. 856, 33-47. doi: 10.1111/j.1749-6632.1998.tb 08310.x

Lam, J., Taylor, V., Islam, S., Hao, Y., and Kocíncová, D. (2011). Genetic and functional diversity of Pseudomonas aeruginosa lipopolysaccharide. Front. Microbiol. 2:118. doi: 10.3389/fmicb.2011.00118

Latino, L., Caroff, M., and Pourcel, C. (2017). Fine structure analysis of lipopolysaccharides in bacteriophage-resistant Pseudomonas aeruginosa PAO1 mutants. Microbiology (Reading) 163, 848-855. doi: 10.1099/mic.0. 000476

Lau, G. W., Hassett, D. J., Ran, H., and Kong, F. (2004). The role of pyocyanin in Pseudomonas aeruginosa infection. Trends Mol. Med. 10, 599-606. doi: 10.1016/j.molmed.2004.10.002

Lee, J., and Zhang, L. (2015). The hierarchy quorum sensing network in Pseudomonas aeruginosa. Protein Cell 6, 26-41. doi: 10.1007/s13238-0140100-x

Li, L.-L., Malone, J. E., and Iglewski, B. H. (2007). Regulation of the Pseudomonas aeruginosa quorum-sensing regulator VqsR. J. Bacteriol. 189, 4367-4374. doi: 10.1128/JB.00007-07

Lin, H.-C., Chen, Y.-J., Wei, Y.-H., Lin, H.-A., Chen, C.-C., Liu, T.-F., et al. (2021). Lactic acid fermentation is required for NLRP3 inflammasome activation. Front. Immunol. 12:630380. doi: 10.3389/fimmu.2021. 630380

Lo Sciuto, A., Cervoni, M., Stefanelli, R., Spinnato, M. C., Di Giamberardino, A., Mancone, C., et al. (2019). Genetic basis and physiological effects of lipid a hydroxylation in Pseudomonas aeruginosa PAO1. Pathogens 8:291. doi: 10. 3390/pathogens 8040291
McIsaac, S. M., Stadnyk, A. W., and Lin, T. J. (2012). Toll-like receptors in the host defense against Pseudomonas aeruginosa respiratory infection and cystic fibrosis. J. Leukoc Biol. 92, 977-985. doi: 10.1189/jlb.0811410

Mirzaei, A., Hedayati, M., Rahbar, M., and Rastegar, H. (2011). A simple method for non phenolic extraction of lipopolysaccharide from Salmonella typhimurium and Salmonella enteritidis with high purity and pyrogenicity in rat. Sci. Res. Essays 6, 1001-1005.

Moradali, M. F., Ghods, S., and Rehm, B. H. A. (2017). Pseudomonas aeruginosa lifestyle: a paradigm for adaptation, survival, and persistence. Front. Cell Infect. Microbiol. 7:39. doi: 10.3389/fcimb.2017.00039

Moser, C., Jensen, P. Ø, Thomsen, K., Kolpen, M., Rybtke, M., Lauland, A. S., et al. (2021). Immune responses to Pseudomonas aeruginosa biofilm infections. Front. Immunol. 12:625597. doi: 10.3389/fimmu.2021.625597

Panayidou, S., Georgiades, K., Christofi, T., Tamana, S., Promponas, V. J., and Apidianakis, Y. (2020). Pseudomonas aeruginosa core metabolism exerts a widespread growth-independent control on virulence. Sci. Rep. 10:9505. doi: 10.1038/s41598-020-66194-4

Pier, G. B. (2007). Pseudomonas aeruginosa lipopolysaccharide: a major virulence factor, initiator of inflammation and target for effective immunity. Int. J. Med. Microbiol. IJMM 297, 277-295. doi: 10.1016/j.ijmm.2007.03.012

Reis, R. S., Pereira, A. G., Neves, B. C., and Freire, D. M. G. (2011). Gene regulation of rhamnolipid production in Pseudomonas aeruginosa - a review. Bioresour. Technol. 102, 6377-6384. doi: 10.1016/j.biortech.2011.03.074

Rossi, E., La Rosa, R., Bartell, J. A., Marvig, R. L., Haagensen, J. A. J., Sommer, L. M., et al. (2021). Pseudomonas aeruginosa adaptation and evolution in patients with cystic fibrosis. Nat. Rev. Microbiol. 19, 331-342. doi: 10.1038/s41579-02000477-5

Sambrook, J. R. (2001). Molecular Cloning: a Laboratory Manual, 3rd Edn. Harbor, NY: Cold Spring Harbor Laboratory.

Simpson, B. W., and Trent, M. S. (2019). Pushing the envelope: LPS modifications and their consequences. Nat. Rev. Microbiol. 17, 403-416. doi: 10.1038/s41579019-0201-x

Stiernagle, T. (2006). Maintenance of C. Elegans. WormBook, ed. The C. elegans Research Community, WormBook, doi: 10.1895/wormbook.1. 101.1

Stover, C. K., Pham, X. Q., Erwin, A. L., Mizoguchi, S. D., Warrener, P., Hickey, M. J., et al. (2000). Complete genome sequence of Pseudomonas aeruginosa PAO1, an opportunistic pathogen. Nature 406, 959-964. doi: 10.1038/350 23079

Thi, M. T. T., Wibowo, D., and Rehm, B. H. A. (2020). Pseudomonas aeruginosa biofilms. Int. J. Mol. Sci. 21:8671. doi: 10.3390/ijms21228671

Toll-Riera, M., San Millan, A., Wagner, A., and MacLean, R. C. (2016). The genomic basis of evolutionary innovation in Pseudomonas aeruginosa. PLoS Genet 12:e1006005. doi: 10.1371/journal.pgen.1006005

Valentini, M., Gonzalez, D., Mavridou, D. A. I., and Filloux, A. (2018). Lifestyle transitions and adaptive pathogenesis of Pseudomonas aeruginosa. Curr. Opin. Microbiol. 41, 15-20. doi: 10.1016/j.mib.2017.11.006

Vital-Lopez, F. G., Reifman, J., and Wallqvist, A. (2015). Biofilm formation mechanisms of Pseudomonas aeruginosa predicted via genome-scale kinetic models of bacterial metabolism. PLoS Comput. Biol. 11:e1004452. doi: 10.1371/ journal.pcbi.1004452

Winson, M. K., Swift, S., Fish, L., Throup, J. P., Jorgensen, F., Chhabra, S. R., et al. (1998). Construction and analysis of luxCDABE-based plasmid sensors for investigating $\mathrm{N}$-acyl homoserine lactone-mediated quorum sensing. FEMS Microbiol. Lett. 163, 185-192. doi: 10.1111/j.1574-6968.1998. tb13044.x

Wong, S. M., and Mekalanos, J. J. (2000). Genetic footprinting with marinerbased transposition in Pseudomonas aeruginosa. Proc. Natl. Acad. Sci. U.S.A. 97, 10191-10196. doi: 10.1073/pnas.97.18.10191

Zarzycki-Siek, J., Norris, M. H., Kang, Y., Sun, Z., Bluhm, A. P., McMillan, I. A., et al. (2013). Elucidating the Pseudomonas aeruginosa fatty acid degradation pathway: identification of additional fatty acyl-CoA synthetase homologues. PLoS One 8:e64554. doi: 10.1371/journal.pone. 0064554

Zasłona, Z., Pålsson-McDermott, E. M., Menon, D., Haneklaus, M., Flis, E., Prendeville, H., et al. (2017). The induction of pro-IL-1 $\beta$ by lipopolysaccharide requires endogenous prostaglandin $\mathrm{E}_{2}$ production. J. Immunol. 198:3558. doi: 10.4049/jimmunol.1602072 
Zhang, Y., and Miller, R. M. (1992). Enhanced octadecane dispersion and biodegradation by a Pseudomonas rhamnolipid surfactant (biosurfactant). Appl. Environ. Microbiol. 58, 3276-3282. doi: 10.1128/AEM.58.10.32763282.1992

Conflict of Interest: The authors declare that the research was conducted in the absence of any commercial or financial relationships that could be construed as a potential conflict of interest.

Publisher's Note: All claims expressed in this article are solely those of the authors and do not necessarily represent those of their affiliated organizations, or those of the publisher, the editors and the reviewers. Any product that may be evaluated in this article, or claim that may be made by its manufacturer, is not guaranteed or endorsed by the publisher.

Copyright (C) 2021 Martínez-Alcantar, Orozco, Díaz-Pérez, Villegas, Reyes-De la Cruz, García-Pineda and Campos-García. This is an open-access article distributed under the terms of the Creative Commons Attribution License (CC BY). The use, distribution or reproduction in other forums is permitted, provided the original author(s) and the copyright owner(s) are credited and that the original publication in this journal is cited, in accordance with accepted academic practice. No use, distribution or reproduction is permitted which does not comply with these terms. 